\title{
WATER RESOURCES OF THE CLINTON RIVER BASIN, SOUTHEASTERN MICHIGAN
}

\author{
By \\ J. O. Nowlin
}

\section{PHYSIOGRAPHIC DATA FOR SELECTED LOCATIONS IN THE CLINTON RIVER BASIN}

The following table summarizes some physiographic data for selected locations on streams in the Clinton River basin. Selected locations include stream confluences, highway crossings, towns, gaging stations, dams, and other points of general interest. Data tabulated include drainage areas, stream miles, altitudes, and average slopes.

For drainage area deterninations, basin divides were delineated on $71 / 2$-minute topographic maps, scale $1: 24,000$, and areas planimetered in accordance with the standards set forth by the Committee on Hydrology, Water Resources Council (formerly Subcommittee on Hydrology, Interagency Committee on Water Resources). Similarly, river miles were determined in accordance with the Committee's standards. Mile zero is considered to be the mouth of the stream. The stream source is considered to be the upper end of the stream, whether or not it is perennial. A defined channel which may be inferred from the topographic maps to carry water intermittently is, therefore, included in the stream length. A stream which begins in name at the confluence of two tributaries is considered to be a continuation of the longer tributary and to have as its source the source of that tributar:'. Its average slope is determined by treating the stream and tributary as one. Paint Creek, for example, is consideret to be the continuation of Paint Creek Drain. In the table, Paint Creek Drain has been parenthetically noted as being the headwaters of Paint Creek.

Altitudes for site and source represent the al titude of the water surface at approximately normal rive- stage. The datum is mean sea level. Average slopes were computed from the mileage between site and sourse and the difference in altitudes.

Streams are listed in a downstream direction along the main stem; tributary streams are entered above the next main-stem location. A similar order is followed for listing streams of first rank, second rank, and other ranks. To indicate the rank and the stream to which it is tributary, the stream name is indented; each indentation represents one rank. The stream rank is further identified by numbers in the column heading with stream name and location. Number 1 applies to Clinton River. Principal tributaries are under number 2 , their tributaries under numt er 3 , and so on.

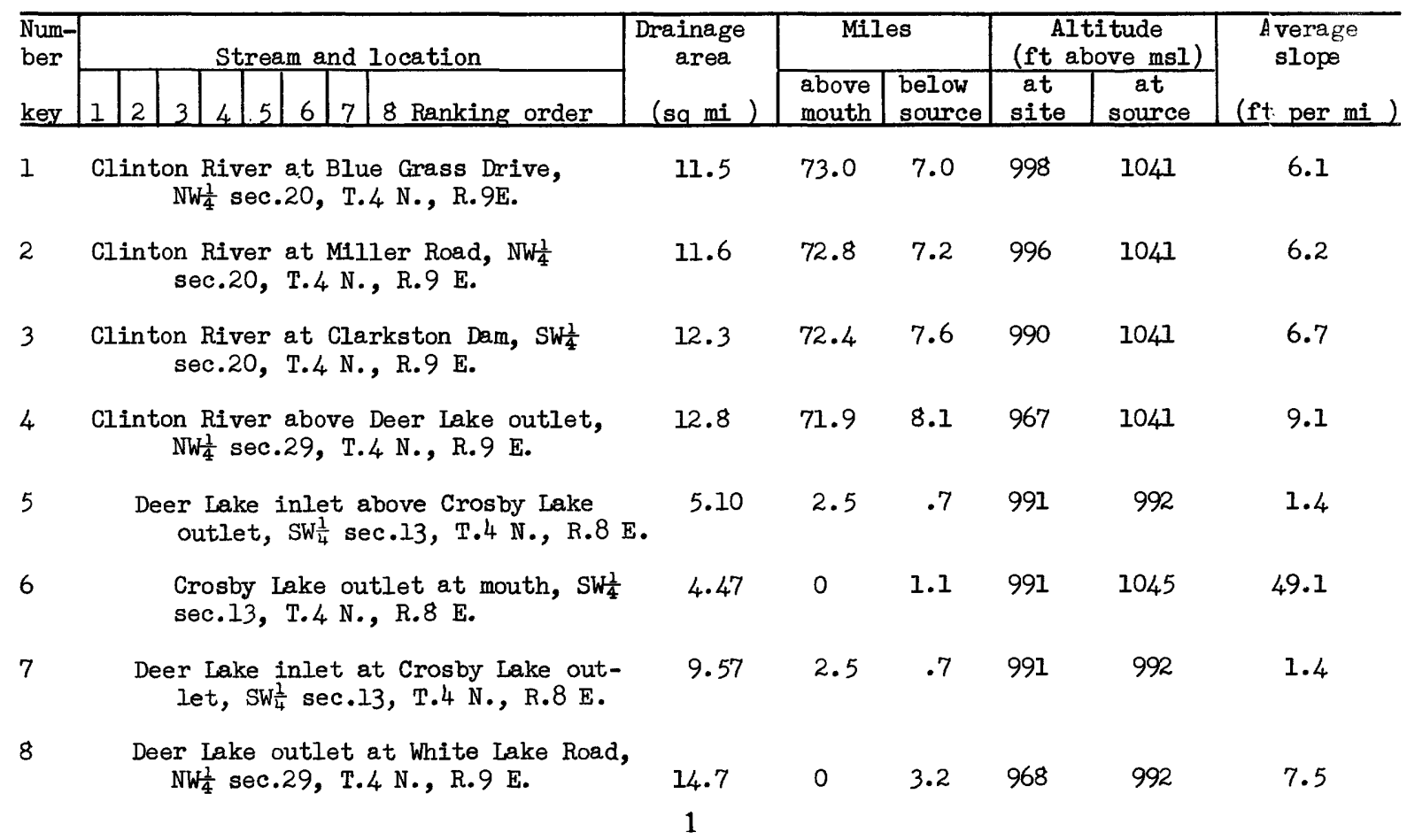


PHYSIOGRAPHIC DATA FOR SETECTED LOCATIONS IN THE CLINTON RIVER BASIN--CONTINUED

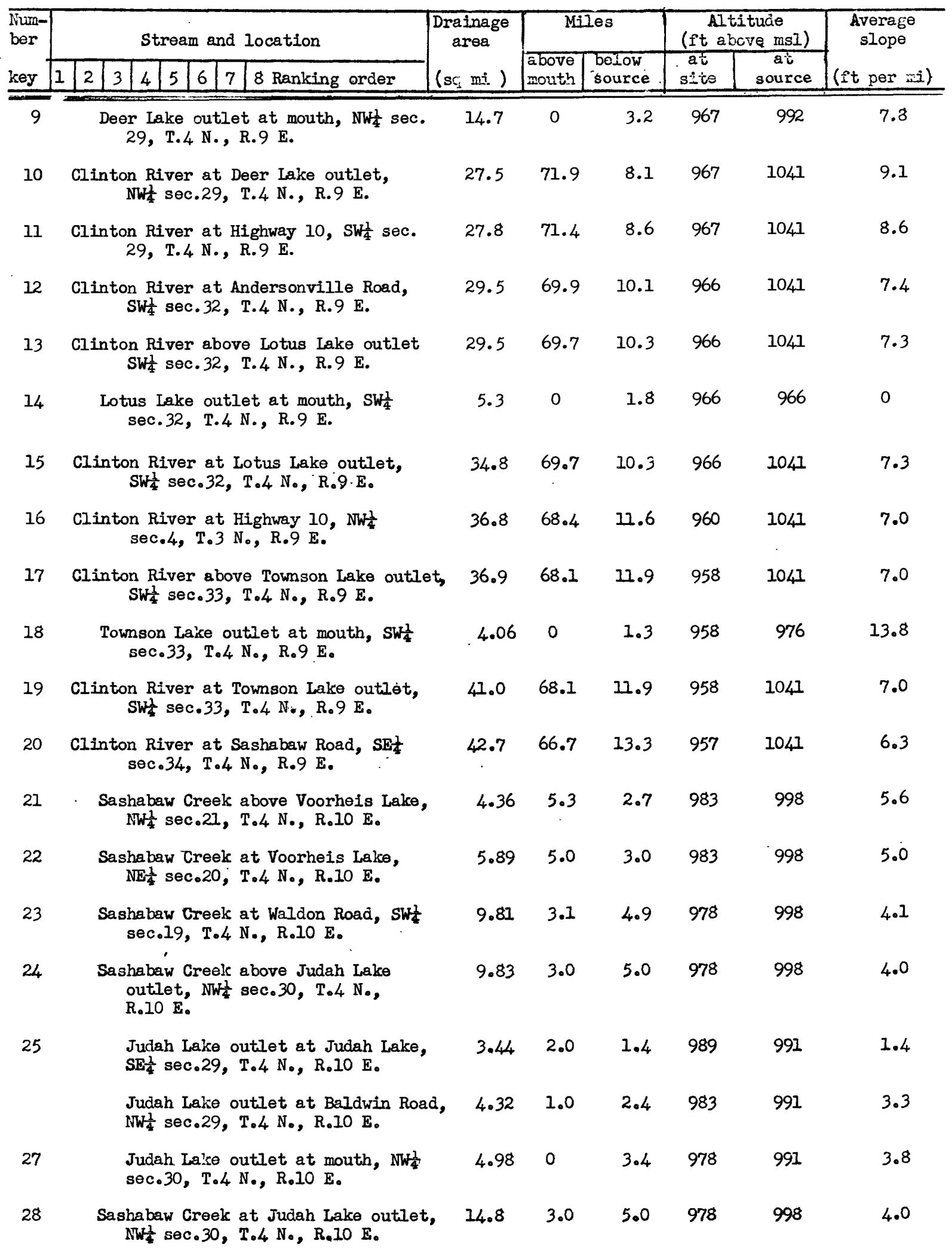


PHYSIOGRAPHIC DATA FOR SELECTED LOCATIONS IN THE CLINTON RIVER BASIN--CONTINUED

\begin{tabular}{|c|c|c|c|c|c|c|c|}
\hline $\begin{array}{l}\text { Num- } \\
\text { ber }\end{array}$ & Stream and location & $\begin{array}{l}\text { Drainage } \\
\text { area }\end{array}$ & $\mathrm{Mil}$ & & $\begin{array}{r}\mathrm{A}] \\
\text { Let } 8\end{array}$ & $\begin{array}{l}\text { itude } \\
\text { ove msI) }\end{array}$ & $\begin{array}{l}\text { Aver:age } \\
\text { slope }\end{array}$ \\
\hline key & \begin{tabular}{|l|l|l|l|l|l|l|l|l|}
1 & 2 & 3 & 4 & 5 & 6 & 7 & 8 Ranking order \\
\end{tabular} & & $\begin{array}{l}\text { above } \\
\text { mouth }\end{array}$ & $\begin{array}{l}\text { below } \\
\text { source }\end{array}$ & $\begin{array}{l}\text { at } \\
\text { site }\end{array}$ & $\begin{array}{c}\text { at } \\
\text { source }\end{array}$ & (ft per mi ) \\
\hline 29 & $\begin{array}{l}\text { Sashabaw Creek above unnamed } \\
\text { tributary, } \mathrm{SE}_{4}^{\frac{1}{4}} \text { sec. } 26, \text { T. } 4 \text { N., } \\
\text { R.9 E. }\end{array}$ & 16.0 & 1.4 & 6.6 & 971 & 998 & 4.1 \\
\hline 30 & $\begin{array}{l}\text { Unnamed tributary at mouth, SE } \frac{1}{4} \\
\text { sec.26, T. } 4 \text { N., R.9 E. }\end{array}$ & 4.33 & 0 & 2.2 & 971 & 1048 & 35.0 \\
\hline 31 & $\begin{array}{l}\text { Sashabaw Creek at unnamed tributary } \\
\text { SE-1 sec.26, T. } 4 \text { N., R. } 9 \text { E. }\end{array}$ & 20.4 & 1.4 & 6.6 & 971 & 998 & 4.1 \\
\hline 32 & $\begin{array}{l}\text { Sashabaw Creek at Maybee Road (gage } \\
\text { 04-1608), SE } \frac{1}{4} \text { sec.26, T.4 N., } \\
\text { R.9 E. }\end{array}$ & 20.9 & 1.0 & 7.0 & 966 & 998 & 4.6 \\
\hline 33 & $\begin{array}{l}\text { Sashabaw Creek at mouth, SE- sec. } 35 \\
\text { T. } 4 \text { N., R. } 9 \text { E. }\end{array}$ & 523.4 & 0 & 8.0 & 957 & 998 & 5.1 \\
\hline 34 & $\begin{array}{c}\text { Clinton River at Walton Boulevard } \\
\text { NE⿺ sec.11, T. } 3 \text { N., R.9 E. }\end{array}$ & 68.8 & $64 \cdot 5$ & 15.5 & 950 & 1041 & 5.9 \\
\hline 35 & $\begin{array}{l}\text { Clinton River at Highway } 10 \\
\text { SE } \frac{1}{4} \text { sec.10, T.3 N., R.9 E. }\end{array}$ & 74.4 & 63.1 & 16.9 & 949 & 1041 & 5.4 \\
\hline 36 & $\begin{array}{l}\text { Clinton River at Highland Road M-59 } \\
\text { (gage 04-1.609), NE } \frac{1}{4} \text { sec.2l, } \\
\text { T.3 N., R.9 E. }\end{array}$ & 79.2 & 60.8 & 19.2 & 940 & 1041 & 5.3 \\
\hline 37 & $\begin{array}{c}\text { Clinton River above unnamed tributary, } \\
\text { NE⿺ sec. } 29 \text {, T.3 N., R. } 9 \text { E. }\end{array}$ & 79.9 & 59.0 & 21.0 & 937 & 1041 & 4.9 \\
\hline 38 & $\begin{array}{l}\text { Unnamed tributary at mouth, NEl } \frac{1}{4} \\
\text { sec. } 29 \text {, T. } 3 \text { N., R. } 9 \text { E. }\end{array}$ & 2.08 & 0 & 2.0 & 937 & 968 & 15.5 \\
\hline 39 & $\begin{array}{c}\text { Clinton River at unnamed tributary } \\
\text { NEt sec. } 29, \text { T. } 3 \text { N., R. } 9 \text { E. }\end{array}$ & 82.0 & 59.0 & 21.0 & 937 & 1041 & 4.9 \\
\hline 40 & $\begin{array}{l}\text { Clinton River at Cooley Road, NW } \frac{1}{4} \\
\text { sec.33, T. } 3 \text { N., R.9 E. }\end{array}$ & 86.4 & 57.2 & 22.8 & 930 & 1041 & 4.9 \\
\hline 41 & $\begin{array}{l}\text { Elizabeth Lake outlet at Elizabeth } \\
\text { Lake Road, NWt sec. } 34 \text {, T. } 3 \text { N., } \\
\text { R. } 9 \text { E. }\end{array}$ & 2.40 & 0 & 0 & 931 & 931 & 0 \\
\hline 42 & $\begin{array}{l}\text { Pine Lake outlet at Orchard Lake } \\
\text { Road, NW } \frac{1}{4} \text { sec.14, T.2 N., } \\
\text { R. } 9 \text { E. }\end{array}$ & 1.82 & 0 & 0 & 932 & 932 & 0 \\
\hline 43 & $\begin{array}{l}\text { Clinton River at Cass Lake outlet, SEt } \\
\text { sec. } 35 \text {, T. } 3 \text { N., R. } 9 \text { E. }\end{array}$ & 99.6 & 53.4 & 26.6 & 929 & 1041 & 4.2 \\
\hline 44 & $\begin{array}{l}\text { Geneva Lake outlet at Elizabeth } \\
\text { Lake Road, NE } \frac{1}{4} \text { sec.26, T.3 N., } \\
\text { R. } 9 \text { E. }\end{array}$ & 2.64 & .7 & 1.9 & 929 & 947 & 9.5 \\
\hline 45 & $\begin{array}{l}\text { Geneva Lake outlet at mouth, } \mathrm{NE}_{4}^{\frac{1}{4}} \\
\text { sec. } 35, \text { T. } 3 \text { N., R. } 9 \mathrm{E} \text {. }\end{array}$ & 3.15 & 0 & 2.6 & 928 & 947 & 7.3 \\
\hline 46 & $\begin{array}{l}\text { Clinton River at Telegraph Road } \\
\text { SW } \frac{1}{4} \text { sec. } 31 \text {, T. } 3 \text { N., R. } 10 \text { E. }\end{array}$ & 105 & 51.7 & 28.3 & 928 & 1041 & 4.0 \\
\hline
\end{tabular}


PHYSIOGRAPHIC DATA FOR SELECTED LOCATIONS IN THE CLINTON RIVER BASTN-CONTINUED

\begin{tabular}{|c|c|c|c|c|c|c|c|}
\hline \multirow{2}{*}{$\begin{array}{l}\text { Num- } \\
\text { ber } \\
\text { key }\end{array}$} & Stream and location & \multirow{2}{*}{$\begin{array}{c}\text { Drainage } \\
\text { area } \\
(\mathrm{sq} \mathrm{mi})\end{array}$} & \multicolumn{2}{|c|}{ Miles } & \multicolumn{2}{|c|}{$\begin{array}{c}\text { Altitude } \\
\text { (ft above msl) }\end{array}$} & \multirow{2}{*}{$\begin{array}{c}\text { Average } \\
\text { slope } \\
\text { (ft per mi) }\end{array}$} \\
\hline & \begin{tabular}{|l|l|l|ll|ll|}
3 & 4 & 5 & 6 & 7 & 8 & Ranking order \\
\end{tabular} & & $\begin{array}{r}\text { above } \\
\text { mouth } \\
\end{array}$ & $\begin{array}{l}\text { below } \\
\text { source }\end{array}$ & $\begin{array}{l}\text { at } \\
\text { site } \\
\end{array}$ & $\begin{array}{c}\text { at } \\
\text { source }\end{array}$ & \\
\hline 47 & $\begin{array}{c}\text { Clinton River at Dawsons Mill Pond, } \\
\text { SW } \frac{1}{4} \text { sec.31, T.3 N., R.10 E. }\end{array}$ & 107 & 50.8 & 29.2 & 923 & 1041 & 4.0 \\
\hline 48 & $\begin{array}{c}\text { Clinton River above Pontiac Creek, } \\
\text { SE } \frac{1}{4} \text { sec.29, T.3 N., R.10 E. }\end{array}$ & 109 & 47.7 & 32.3 & 910 & 1041 & 4.1 \\
\hline 49 & $\begin{array}{l}\text { Pontiac Creek at Highway 10, } \mathrm{NE}_{4}^{\frac{1}{4}} \\
\text { sec.19, T.3 N., R.10 E. }\end{array}$ & 4.95 & 2.0 & 1.5 & 931 & 940 & 6.0 \\
\hline 50 & $\begin{array}{l}\text { Pontiac Creek at mouth, SE } \frac{1}{4} \text { sec.29, } \\
\text { T.3 N., R.10 E. }\end{array}$ & 7.21 & 0 & 3.5 & 910 & 940 & 8.6 \\
\hline 51 & $\begin{array}{c}\text { Clinton River at Pontiac Creek, } \mathrm{SE}_{\frac{1}{4}} \\
\text { sec.29, T.3 N., R.10 E. }\end{array}$ & 116 & 47.7 & 32.3 & 910 & 1041 & 4.1 \\
\hline 52 & $\begin{array}{c}\text { Clinton River at Pontiac Sewage Plant } \\
\text { NW } \frac{1}{4} \text { sec. } 27 \text {, T. } 3 \text { N., R.10 E. }\end{array}$ & 119 & 45.5 & $34 \cdot 5$ & 878 & 1047 & 4.7 \\
\hline 53 & $\begin{array}{c}\text { Clinton River at Opdyke Road, } \mathrm{SE}_{4}^{1} \\
\text { sec.27, T.3 N., R.10 E. }\end{array}$ & 120 & 33.9 & 36.1 & 859 & 1041 & 5.0 \\
\hline 54 & $\begin{array}{l}\text { Clinton River at Auburn Heights (gage } \\
04-1610 \text { ), NW } \frac{1}{4} \text { sec. } 36, \text { T. } 3 \text { N., } \\
\text { R.10 E. }\end{array}$ & 123 & 42.3 & 37.7 & 850 & 1041 & 5.1 \\
\hline 55 & $\begin{array}{c}\text { Clinton River above Calloway Creek } \\
\mathrm{SE}_{\frac{1}{4}}^{\frac{1}{4}} \text { sec.19, T.3 N., R.11 E. }\end{array}$ & 126 & 39.6 & 40.4 & 808 & 1041 & 5.8 \\
\hline 56 & $\begin{array}{l}\text { Calloway Creek above Calloway } \\
\text { Ditch, SW } \frac{1}{4} \text { sec.10, T.3 N., } \\
\text { R.10 E. }\end{array}$ & 5.38 & 5.6 & 2.3 & 916 & 948 & 13.9 \\
\hline 57 & $\begin{array}{l}\text { Calloway Ditch at mouth, SW- } \frac{1}{4} \\
\text { sec.10, T.3 N., R.10 E. }\end{array}$ & 4.34 & 0 & 4.6 & 916 & 1070 & 33.5 \\
\hline 58 & $\begin{array}{l}\text { Galloway Creek at Calloway Ditch } \\
\text { SW } \frac{1}{4} \text { sec.10, T. } 3 \text { N., R.10 E. }\end{array}$ & 9.72 & 5.6 & 2.3 & 916 & 948 & 13.9 \\
\hline 59 & $\begin{array}{l}\text { Calloway Creek at Walton Boulevard, } \\
\text { SW } \frac{1}{4} \text { sec.10, T.3 N., R.10 E. }\end{array}$ & 9.78 & 5.5 & 2.4 & 915 & 948 & 13.8 \\
\hline 60 & $\begin{array}{l}\text { Calloway Creek at Perry Street, } \mathrm{SE}_{\frac{1}{4}}^{1} \\
\text { sec.15, T.3 N., R.10 E. }\end{array}$ & 10.8 & 4.8 & 3.1 & 906 & 948 & 13.5 \\
\hline 61 & $\begin{array}{l}\text { Calloway Creek at Opdyke Road, NW } \frac{1}{4} \\
\text { sec.23, T.3 N., R.10 E. }\end{array}$ & 12.1 & 3.9 & 4.0 & 900 & 948 & 12.0 \\
\hline 62 & $\begin{array}{l}\text { Calloway Creek at Squirrel Road, } \\
\text { NE } \frac{1}{4} \text { sec.24, T. } 3 \text { N., R.IO E. }\end{array}$ & 14.1 & 1.9 & 6.00 & 840 & 948 & 18.0 \\
\hline 63 & $\begin{array}{l}\text { Calloway Creek above unnamed } \\
\text { tributary, } \mathrm{SE}_{\frac{1}{4}}^{-1} \text { sec.18, T.3 N., } \\
\text { R.Il E. }\end{array}$ & 14.9 & .8 & 7.1 & 824 & 948 & 17.5 \\
\hline 64 & $\begin{array}{l}\text { Unnamed tributary at mouth, } \mathrm{SE}_{4}^{\frac{1}{4}} \\
\text { sec.18, T. } 3 \text { N., R.11 E. }\end{array}$ & 3.00 & 0 & 2.4 & 824 & 953 & 53.8 \\
\hline 65 & $\begin{array}{l}\text { Galloway Creek at unnamed tributary } \\
\text { (Auburn Heights gage 04-16ll), } \\
\text { SE } \frac{1}{4} \text { sec.18, T.3 N., R.11 E. }\end{array}$ & 17.9 & .8 & 7.1 & 824 & 948 & 17.5 \\
\hline
\end{tabular}


PHYSIOGRAPHIC DATA FOR SELECTED LOCATIONS IN THE CLINTON RIVER BASIN--CONTINUED

\begin{tabular}{|c|c|c|c|c|c|c|c|}
\hline $\begin{array}{l}\text { Num- } \\
\text { ber }\end{array}$ & Stream and location & $\begin{array}{c}\text { Drainage } \\
\text { area }\end{array}$ & & & $\begin{array}{r}\mathrm{Al} \\
\text { (ft a }\end{array}$ & $\begin{array}{l}\text { tude } \\
\text { ve msI) }\end{array}$ & $\begin{array}{l}\text { Arerage } \\
\text { slope }\end{array}$ \\
\hline key & \begin{tabular}{l|l|l|l|l|l|l}
2 & 3 & 4 & 5 & 6 & 7 & 8 Ranking order \\
\end{tabular} & $(\mathrm{sg} \mathrm{mi})$ & $\begin{array}{l}\text { above } \\
\text { mouth }\end{array}$ & $\begin{array}{l}\text { below } \\
\text { source }\end{array}$ & $\begin{array}{l}\text { at } \\
\text { site }\end{array}$ & $\begin{array}{c}\text { at } \\
\text { source }\end{array}$ & (ft per mi ) \\
\hline 66 & $\begin{array}{l}\text { Calloway Creek above Miller Lake } \\
\text { outlet, NE-1 sec.19, T.3 N., } \\
\text { R.11 E. }\end{array}$ & 18.0 & .6 & 7.3 & 822 & 948 & 17.3 \\
\hline 67 & 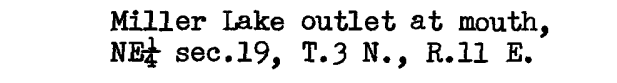 & 1.23 & 0 & 2.2 & 822 & 978 & 70.9 \\
\hline 68 & $\begin{array}{l}\text { Calloway Creek at Miller Lake } \\
\text { outlet, NE⿺ sec.19, T.3 N., } \\
\text { R.Il E. }\end{array}$ & 19.2 & .6 & 7.3 & 822 & 948 & 17.3 \\
\hline 69. & $\begin{array}{l}\text { Calloway Creek at mouth, SE- sec.19 } \\
\text { T.3 N., R.11 E. }\end{array}$ & $9,19.6$ & 0 & 7.9 & 808 & 948 & 17.7 \\
\hline 70 & $\begin{array}{c}\text { Clinton River at Galloway Creek, } \mathrm{SE} \frac{1}{4} \\
\text { sec.19, T.3 N., R.II E. }\end{array}$ & 146 & 39.6 & 40.4 & 808 & 1041 & 5.8 \\
\hline 71 & 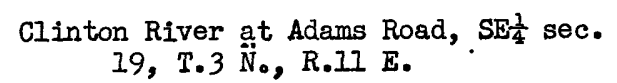 & 146 & $38: 8$ & 41.2 & 796 & 1041 & 5.9 \\
\hline 72 & $\begin{array}{c}\text { Clinton River at Crooks Road, SE } \frac{1}{4} \\
\text { sec.20, T.3 N., R.Il E. }\end{array}$ & 147 & 37.2 & 42.8 & 770 & 1041 & 6.3 \\
\hline 73 & $\begin{array}{l}\text { Clinton River at Rochester Road, NE } \frac{1}{4} \\
\text { sec.15, T.3 N., R.Il E. }\end{array}$ & 152 & 33.9 & 46.1 & 718 & 1041 & 7.0 \\
\hline 74 & $\begin{array}{c}\text { Clinton River above Paint Creek, NWt } \\
\text { sec.14, T.3 N., R.11 E. }\end{array}$ & 152 & 33.6 & 46.4 & 712 & 1041 & 7.1 \\
\hline 75 & $\begin{array}{l}\text { Paint Creek Drain (headwaters of } \\
\text { Paint Creek) at Seymour Lake } \\
\text { Road, SE } \frac{1}{4} \text { sec.26, T.5 N., } \\
\text { R.9 E. }\end{array}$ & 6.61 & 21.9 & 4.3 & 1032. & 1090 & 13.5 \\
\hline 76 & $\begin{array}{l}\text { Paint Creek Drain above Dennis } \\
\text { Lake outlet, SW } W^{\frac{1}{4}} \text { sec. } 5, \mathrm{~T} .4 \mathrm{~N} . \text {, } \\
\text { R.10 E. }\end{array}$ & 12.1 & 19.0 & 7.2 & 1008 & 1090 & 11.4 \\
\hline 77 & $\begin{array}{l}\text { Dennis Lake outlet at mouth, } \\
\text { SW } \frac{1}{4} \text { sec.5, T.4 N., R.10 E. }\end{array}$ & 3.90 & 0 & 1.1 & 1010 & 1020 & 9.1 \\
\hline 78 & $\begin{array}{l}\text { Paint Creek Drain at Dennis Lake } \\
\text { outlet, SW- } \sec .5, \mathrm{~T} .4 \mathrm{~N} . \text {, } \\
\text { R.10 E. }\end{array}$ & 16.0 & 19.0 & 7.2 & 1008 & 2090 & 11.4 \\
\hline 79 & $\begin{array}{l}\text { Paint Creek Drain above unnamed } \\
\text { tributary, NWt sec.4, T.4 N., } \\
\text { R.10 E. }\end{array}$ & 16.6 & 17.5 & 8.7 & 998 & 1090 & 10.6 \\
\hline 80 & 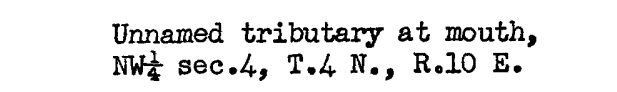 & 1.64 & 0 & .4 & 998 & 1008 & 25.0 \\
\hline 81 & $\begin{array}{l}\text { Paint Creek Drain at unnamed } \\
\text { tributary, NWt sec.4, T.4 N., } \\
\text { R.10 E. }\end{array}$ & 18.3 & 27.5 & 8.7 & 998 & 1090 & 10.6 \\
\hline 82 & $\begin{array}{l}\text { Paint Creek above Indianwood Lake, } \\
\text { SE- } 86.33 \text {, T. } 5 \text { \$., B.10 E. }\end{array}$ & 18.9 & 16.6 & 9.6 & 992 & 1090 & 10.2 \\
\hline
\end{tabular}


PHYSIOGRAPHIC DATA FOR SETECTED LOCATIONS IN THE CLINTON RIVER BASIN-CONTINUED

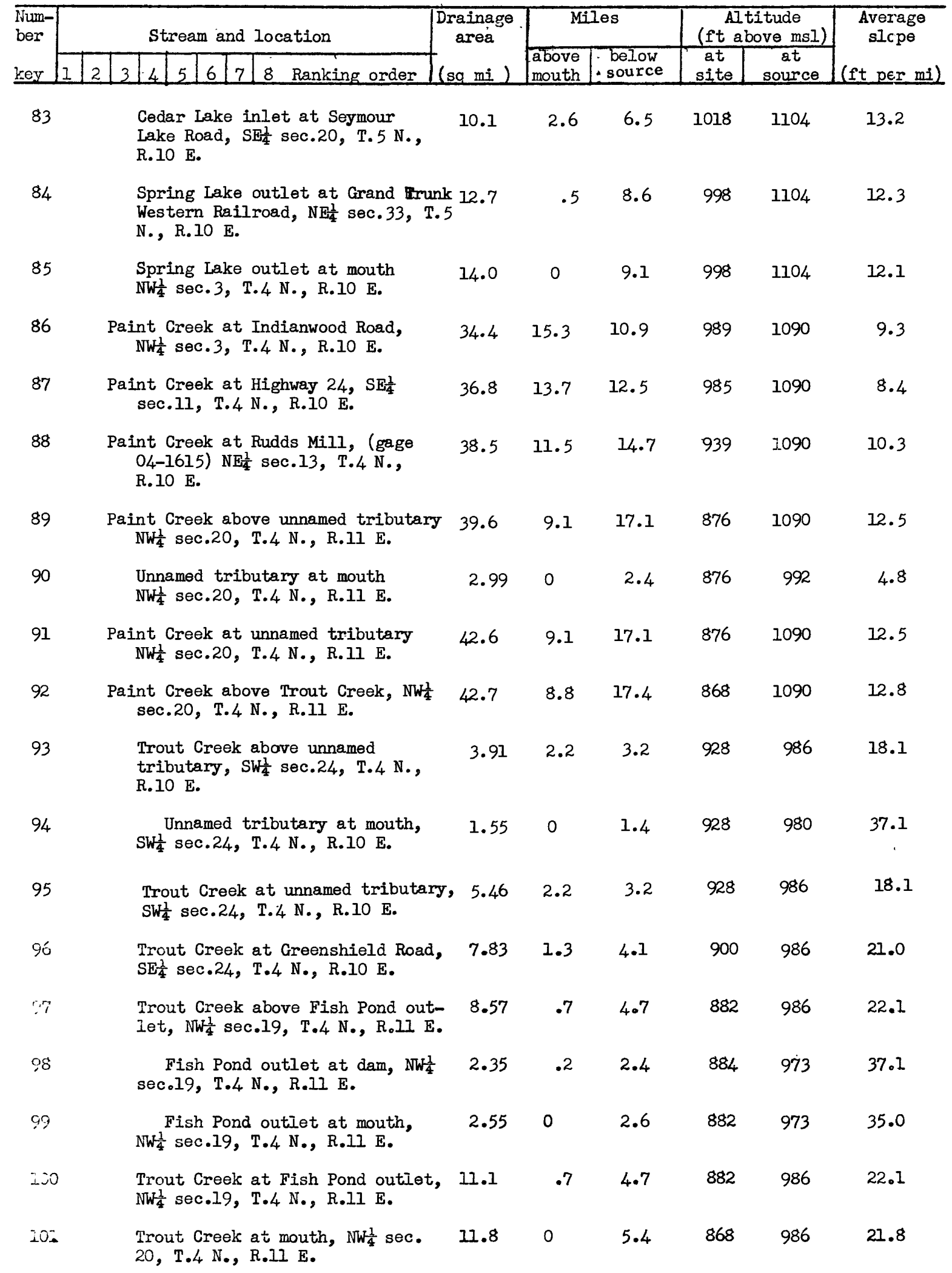


PHYSIOGRAPHIC DATA FOR SELECTED LOCATIONS IN THE CLINTON RIVER BASIN-CONTINUED

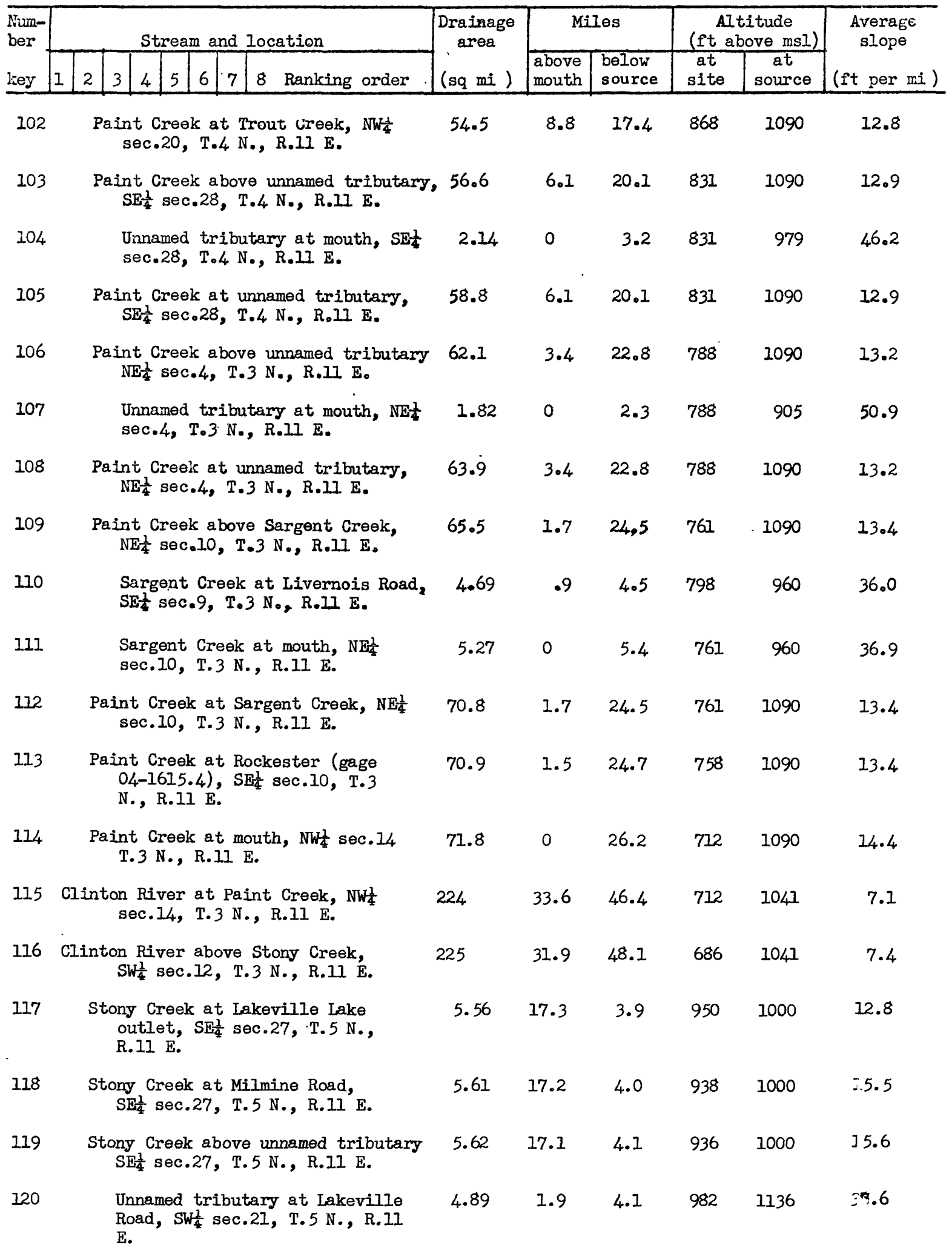


PHYSIOGRAPHIC DATA FOR SELECTED LOCATIONS IN THE CLINTON RIVER BASIN-CONTINUED




PHYSIOGRAPHIC DATA FOR SELECTED IOCATIONS IN THE CLINTON RIVER BASIN-CONTINUED

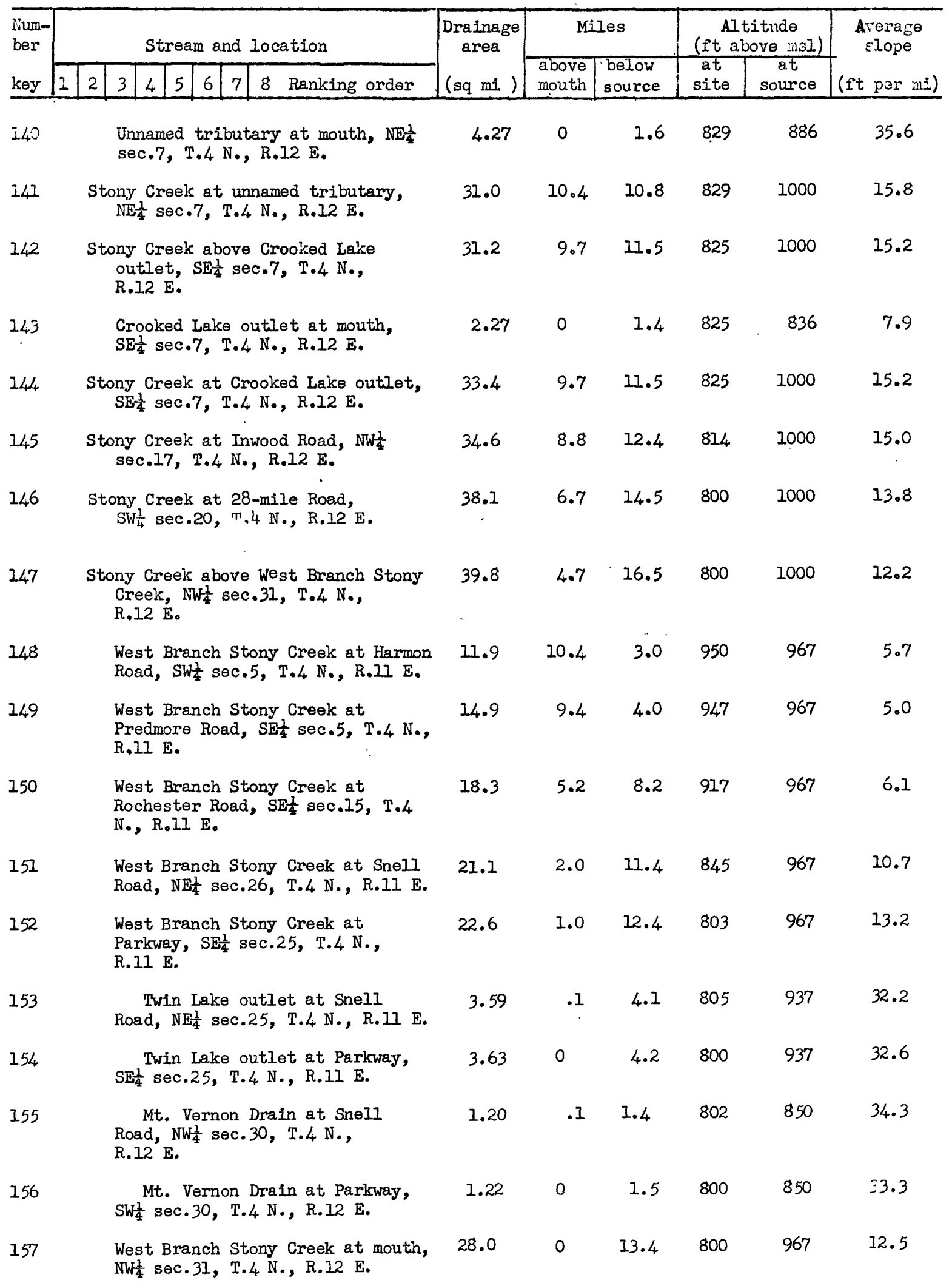


PHYSIOGRAPHIC DATA FOR SELECTED LOGATIONS IN THE CLINTON RIVER BASIN-CONTINUED

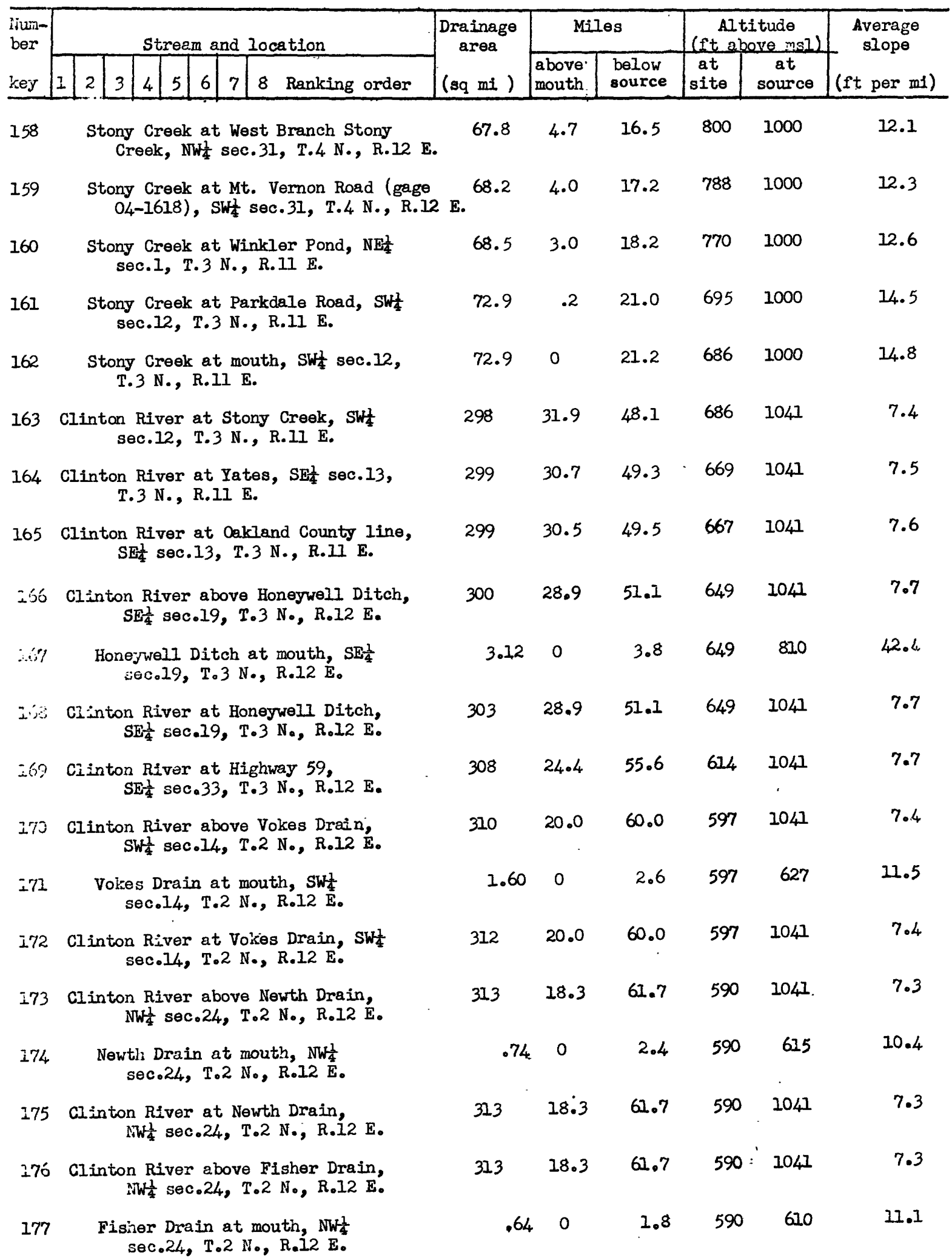


PHYSIOGRAPHIC DATA FOR SETECTED LOCATIONS IN THE CLINTON RIVER BASIN-CONTINUED

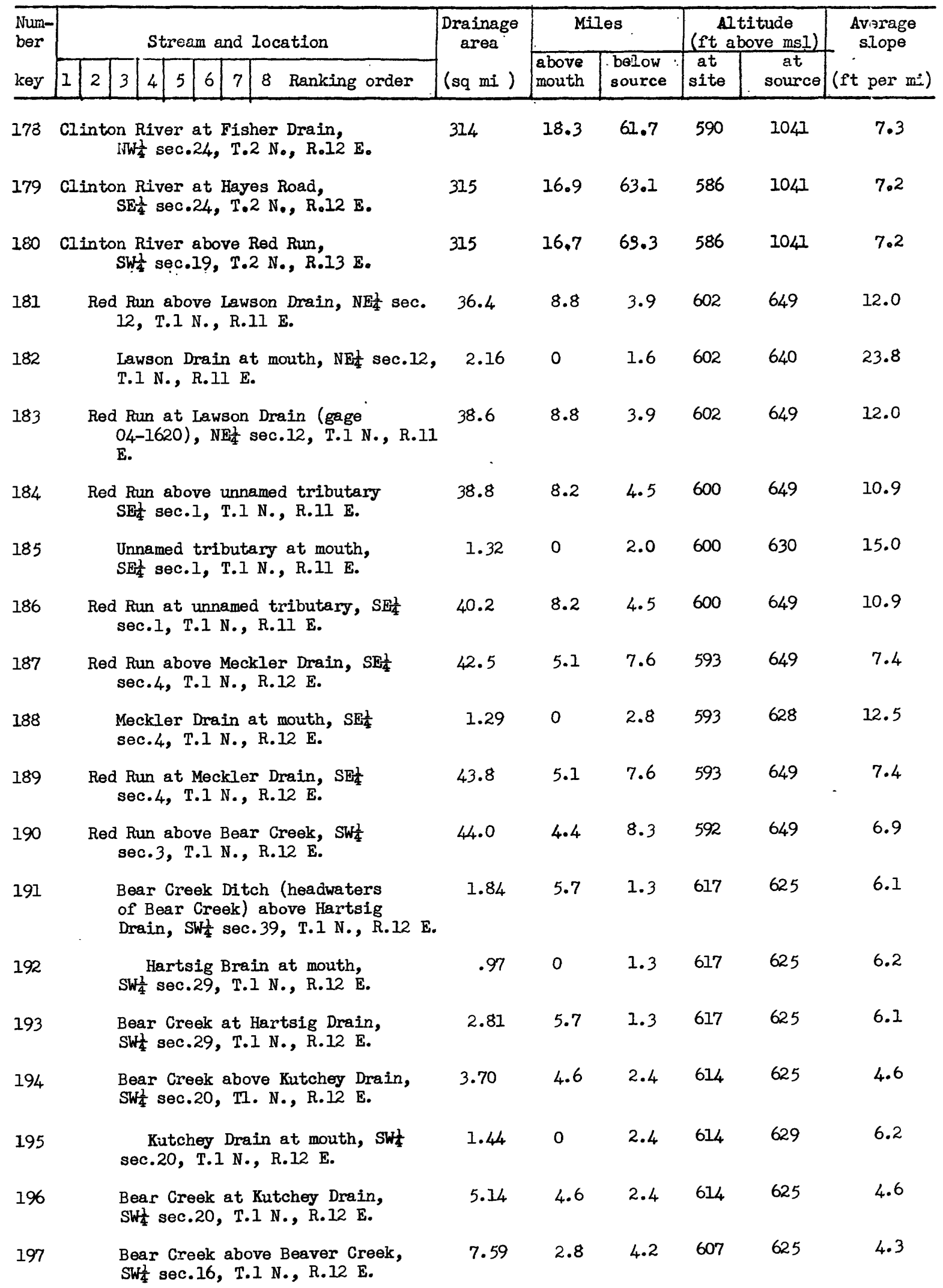


PHYSIOGRAPHIC DATA FOR SELECTED LOCATIONS IN THE CLINTON RIVER BASIN--CONTINUED

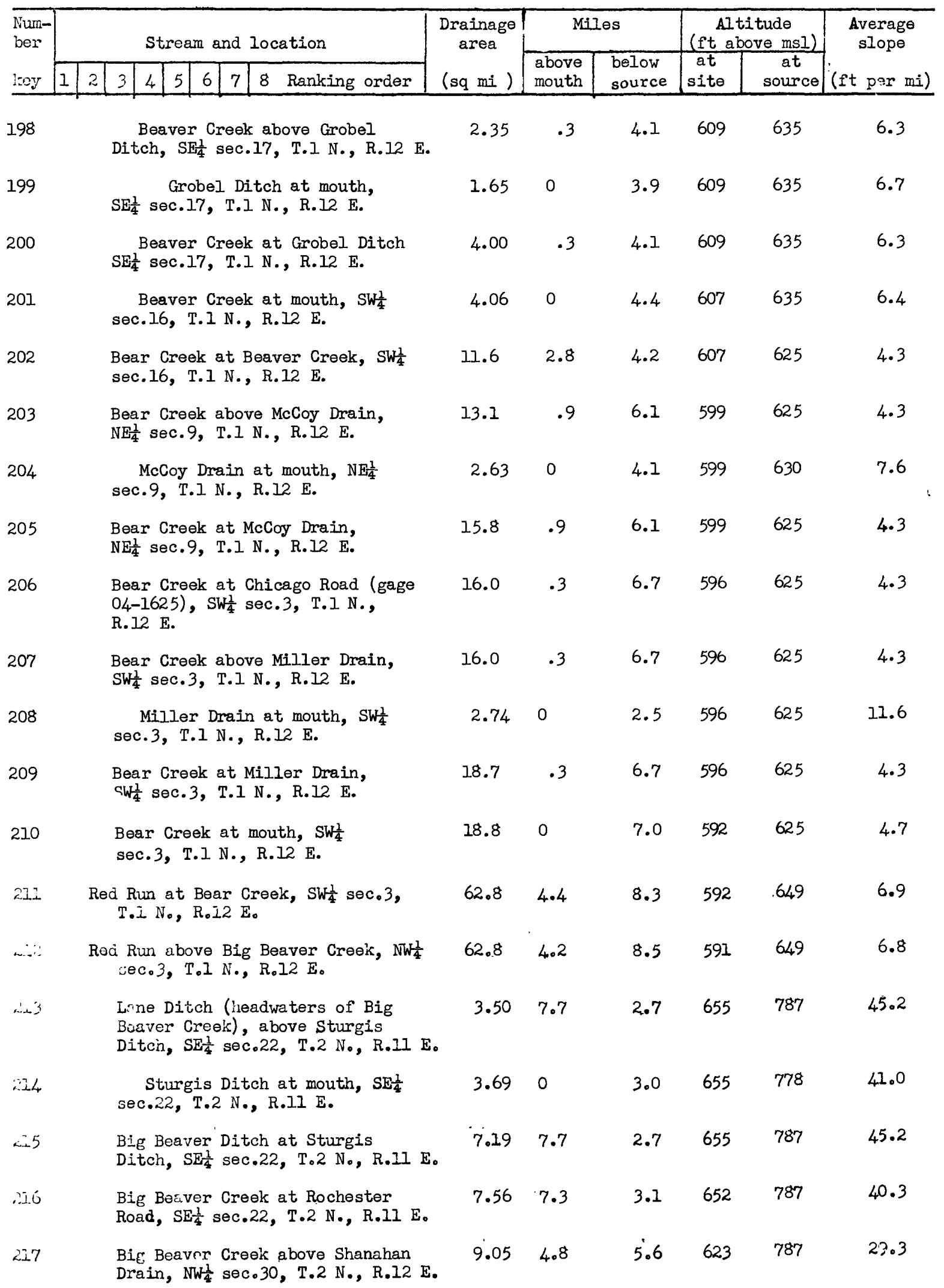


PHYSIOGRAPHIC DATA FOR SETECTED LOCATIONS IN THE CLINTON RIVER BASIN-CONTINUED

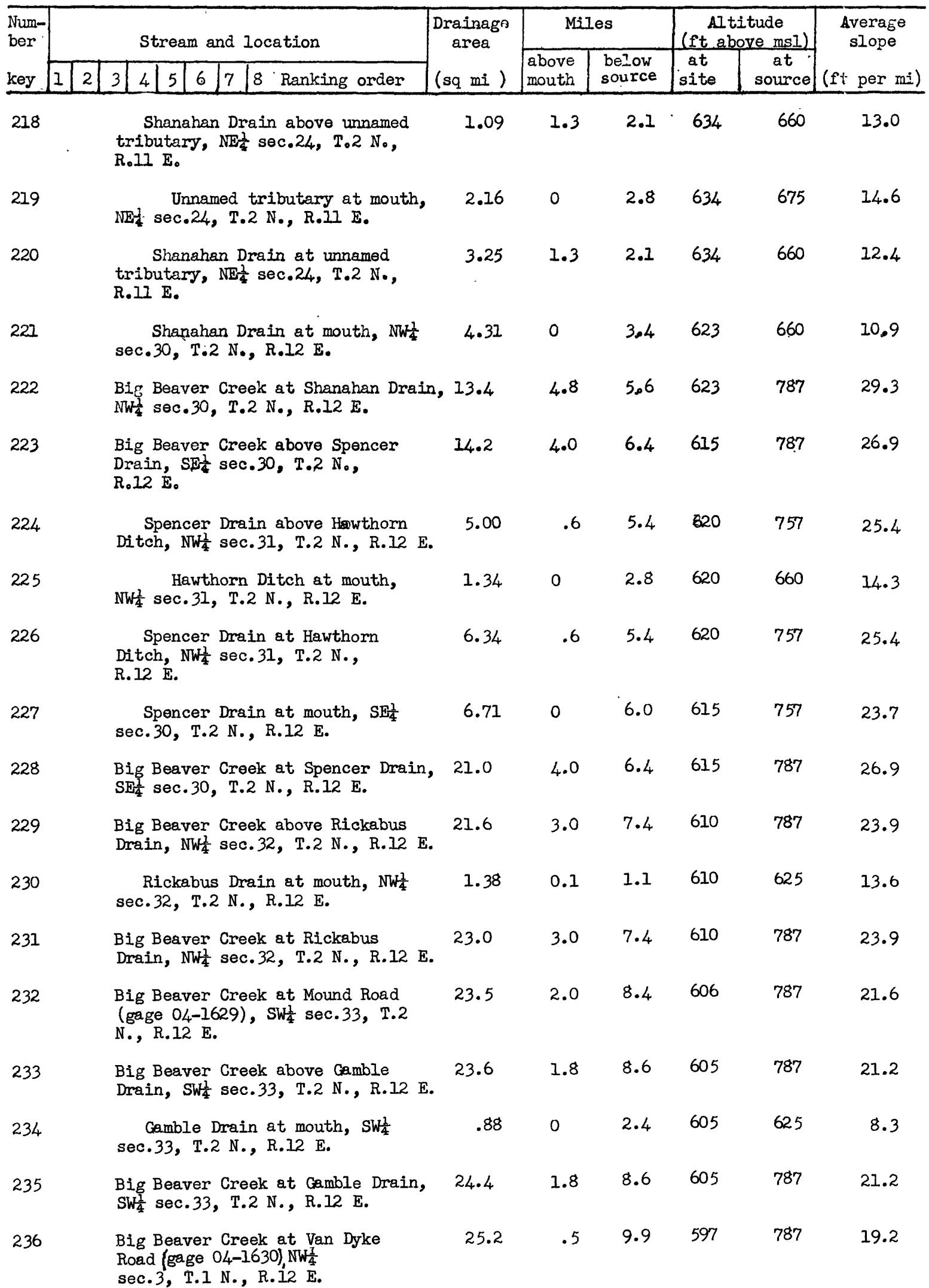


PHYSIOGRAPHIC DATA FOR SELECTED LOCATIONS IN THE CLINTON RIVER BASIN--CONTINUED

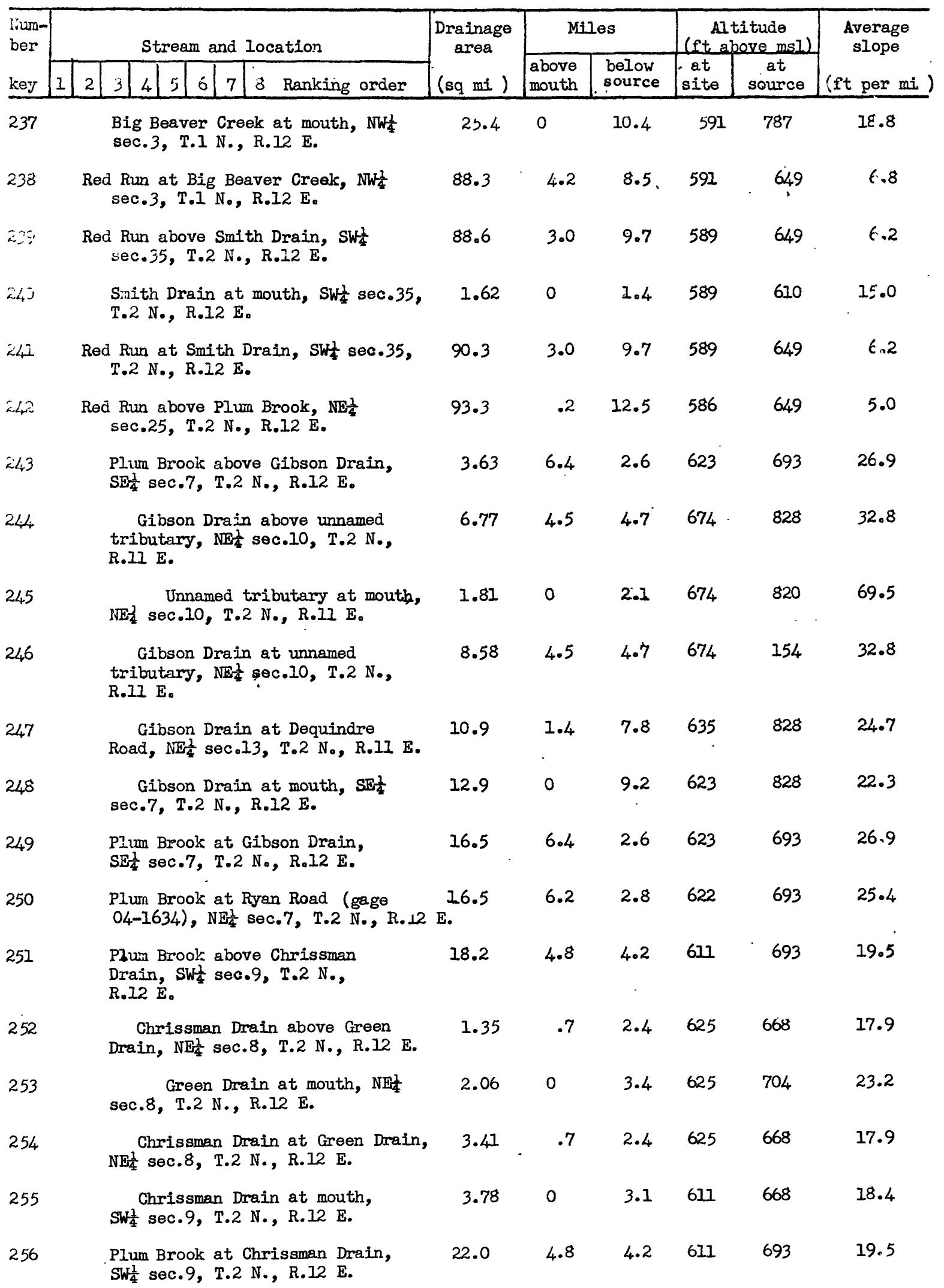


PHYSIOGRAPHIC DATA FOR SELECTED LOCATIONS IN THE CLINTON RIVER BASIN-CONTINUED

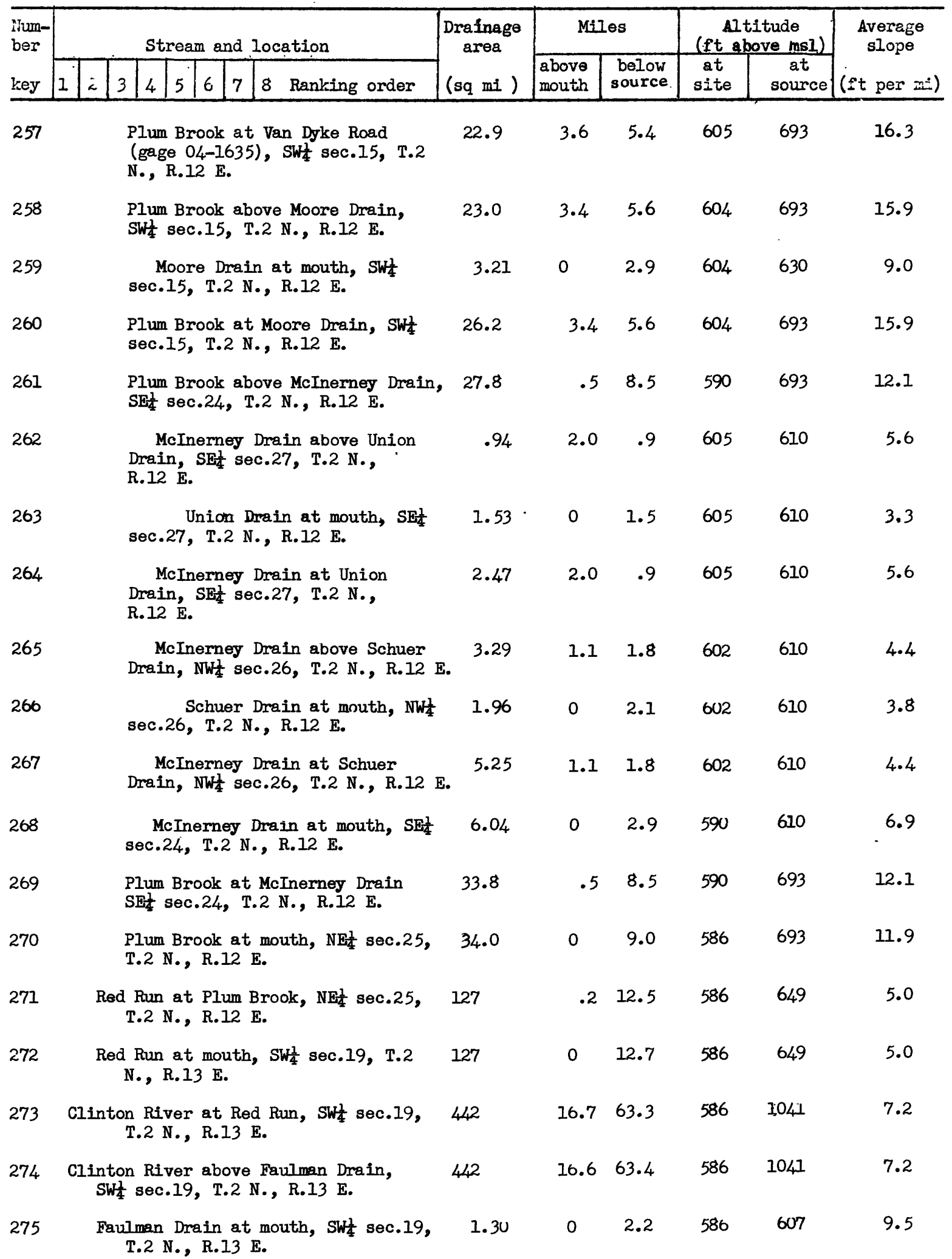


PHISTOGRAPHIC DATA FOR SELECTED LOCATIONS IN THE CLINTON RIVER BASIN-CONTINUED

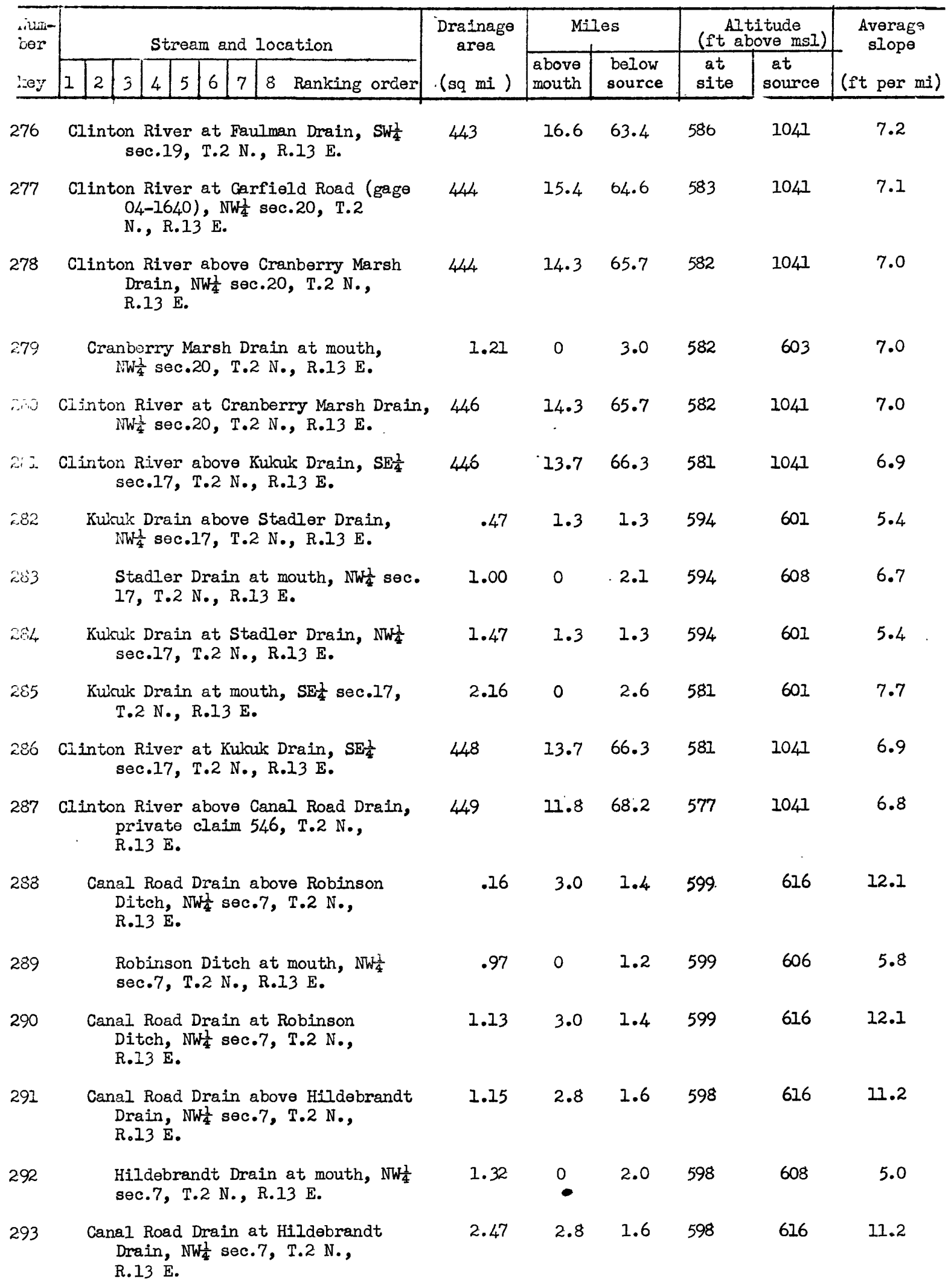


PHYSIOGRAPHIC DATA FOR SELECTED LOCATIONS IN THE CLINTON RIVER BASIN--CONTINUED

\begin{tabular}{|c|c|c|c|c|c|c|c|}
\hline \multirow{2}{*}{$\begin{array}{l}\text { ivum- } \\
\text { ber } \\
\text { i:ey }\end{array}$} & Stream and location & \multirow{2}{*}{$\begin{array}{c}\text { Drainage } \\
\text { area } \\
\left(\mathrm{sq}_{\text {ini }}\right)\end{array}$} & \multicolumn{2}{|c|}{ Miles } & \multicolumn{2}{|c|}{$\begin{array}{l}\text { Altitude } \\
\text { (ft above msl) }\end{array}$} & \multirow{2}{*}{$\begin{array}{c}\text { Averáge } \\
\text { slope } \\
\text { (ft. per in) }\end{array}$} \\
\hline & \begin{tabular}{|l|l|l|l|l|l|l|l|l|}
1 & 2 & 3 & 4 & 5 & 6 & 7 & 8 Ranking order & ( \\
\end{tabular} & & $\begin{array}{l}\text { above: } \\
\text { mouth }\end{array}$ & $\begin{array}{l}\text { below } \\
\text { source }\end{array}$ & $\begin{array}{l}\text { at } \\
\text { site }\end{array}$ & $\begin{array}{c}\text { at } \\
\text { source }\end{array}$ & \\
\hline 294 & $\begin{array}{l}\text { Canal Road Drain at mouth, private } \\
\text { claim 546, T.2 N., R.13 E. }\end{array}$ & 3.60 & 0 & $4 \cdot 4$ & 577 & 616 & 8.9 \\
\hline 295 & $\begin{array}{l}\text { Clinton River at Canal Road Drain, } \\
\text { private claim 546, T.2 N., R. } 13 \\
\text { E. }\end{array}$ & 453 & 11.8 & 68.2 & 577 & 1041 & 6.8 \\
\hline 296 & $\begin{array}{l}\text { Clinton River above North Branch } \\
\text { Clinton River, private claim } \\
546, \text { T.2 N., R.13 E. }\end{array}$ & 453 & 11.0 & 69.0 & 576 & 1041 & 6.7 \\
\hline 297 & $\begin{array}{l}\text { North Branch Clinton River at } \\
\text { Boardman Road, NWt sec.5, T. } 5 \\
\text { N., R.12 E. }\end{array}$ & 2.60 & 40.6 & 2.6 & 880 & 973 & 35.8 \\
\hline 298 & $\begin{array}{l}\text { North Branch Clinton River above } \\
\text { unnamed tributary, SE⿺ sec.28, } \\
\text { T.6 N., R.12 E. }\end{array}$ & 5.57 & 38.8 & $4 \cdot 4$ & 839 & 973 & 30.5 \\
\hline 299 & $\begin{array}{l}\text { Unnamed tributary at mouth, } \\
\text { SE } \frac{1}{4} \text { sec. } 28, \text { T.6 N., R. } 12 \text { E. }\end{array}$ & 1.81 & 0 & 2.6 & 839 & 988 & 57.3 \\
\hline 300 & $\begin{array}{l}\text { North Branch Clinton River at } \\
\text { unnamed tributary, SE-1 sec.28, } \\
\text { T.6 N., R.12 E. }\end{array}$ & 7.38 & 38.8 & 4.4 & 839 & 973 & 30.5 \\
\hline 301 & $\begin{array}{l}\text { North Branch Clinton River above } \\
\text { unnamed tributary, NEt sec.28, } \\
\text { T.6 N., R.12 E. }\end{array}$ & 8.13 & 38.0 & 5.2 & 828 & 973 & 2.7 .9 \\
\hline 302 & $\begin{array}{l}\text { Unnamed tributary at mouth, } \\
\text { NEt sec. } 28, \text { T. } 6 \text { N., R.12 E. }\end{array}$ & 1.43 & 0 & 1.4 & 828 & 870 & 30.0 \\
\hline 303 & $\begin{array}{l}\text { North Branch Clinton River at } \\
\text { unnamed tributary at Highway } \\
53 \text { (gage } 04-1640.1 \text { ), NE } \frac{1}{4} \text { sec.28, } \\
\text { T.6 N., R.12 E. }\end{array}$ & 9.56 & 38.0 & 5.2 & 828 & 973 & 2.7 .9 \\
\hline 304 & $\begin{array}{l}\text { North Branch Clinton River above } \\
\text { unnamed tributary, SE } \frac{1}{4} \text { sec. } 35 \text {, } \\
\text { T.6 N., R.12 E. }\end{array}$ & 11.6 & $34 \cdot 4$ & 8.8 & 779 & 973 & 22.0 \\
\hline 305 & $\begin{array}{l}\text { Unnamed tributary at mouth, } \mathrm{SE} \frac{1}{4} \\
\text { sec.35, T.6 N., R.12 E. }\end{array}$ & 1.67 & 0 & 1.6 & 779 & 808 & 38.1 \\
\hline 306 & $\begin{array}{l}\text { North Branch Clinton River at unnamed } \\
\text { tributary, SE } \frac{1}{4} \text { sec.35, T.6 N., } \\
\text { R.12 E. }\end{array}$ & d 13.2 & $34 \cdot 4$ & 8.8 & 779 & 973 & 2.2 .0 \\
\hline 307 & $\begin{array}{l}\text { North Branch Clinton above unnamed } \\
\text { tributary, SW-1 sec.36, T.6 N., } \\
\text { R.12 E. }\end{array}$ & 13.2 & 34.4 & 8.8 & 779 & 973 & 22.0 \\
\hline 308 & $\begin{array}{l}\text { Unnamed tributary at mouth, SW } \\
\text { sec.36, T.6 N., R.12 E. }\end{array}$ & 3.89 & 0 & 4.4 & 779 & 809 & 6.8 \\
\hline 309 & $\begin{array}{l}\text { North Branch Clinton River at unnamed } \\
\text { tributary (at Lapeer County line), } \\
\text { SW } \frac{1}{4} \text { sec.36, T.6 N., R.12 E. }\end{array}$ & 17.1 & 34.4 & 8.8 & 779 & 973 & 22.0 \\
\hline
\end{tabular}


PHYSIOGRAPHIC DATA FOR SETECTED LOCATIONS IN THE CLINTON RIVER BASIN-CONTINUED

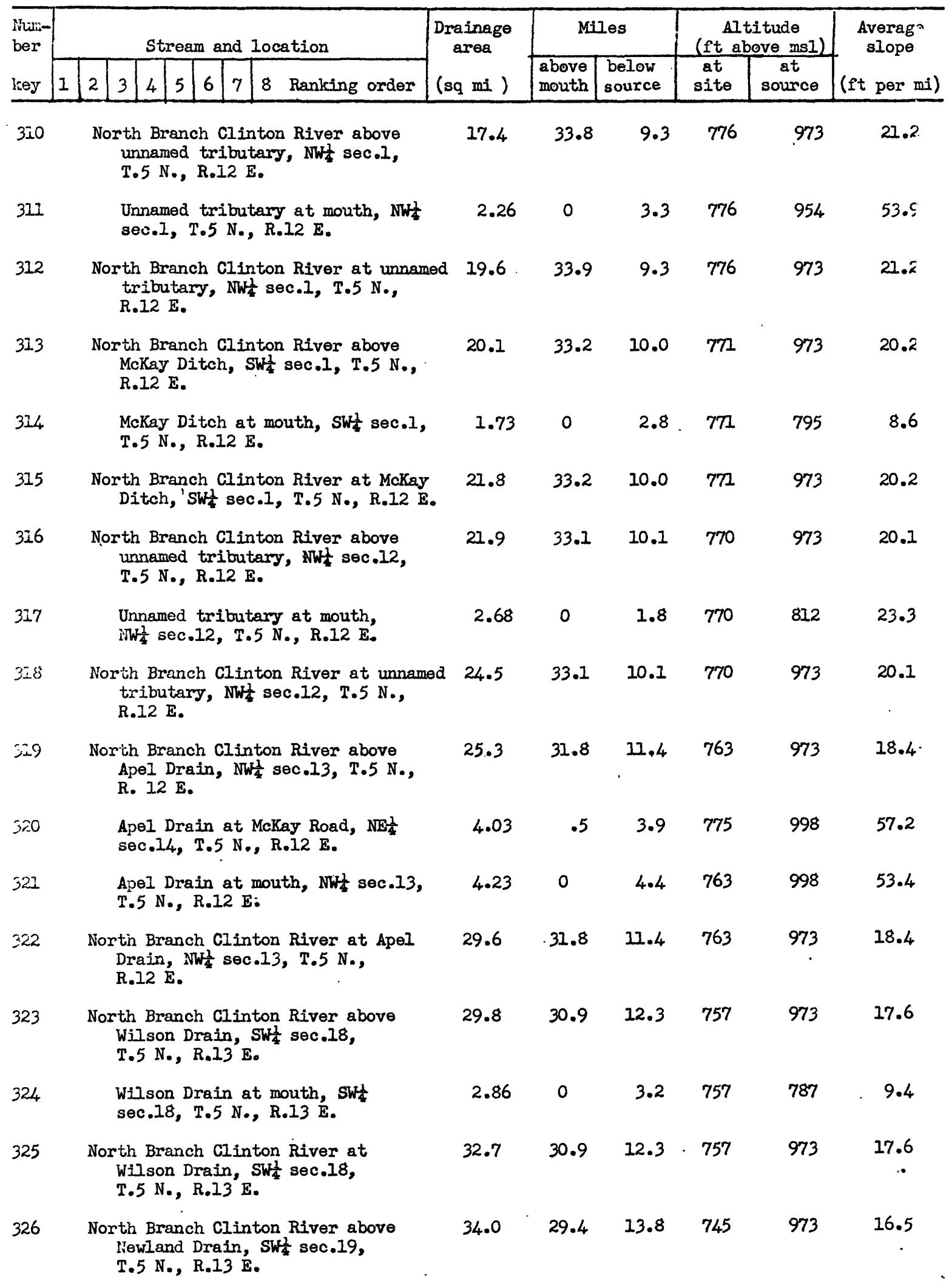


PHYSIOGRAPHIC DATA FOR SELECTED LOCATIONS IN THE CLINTON BASIN-CONTINUED

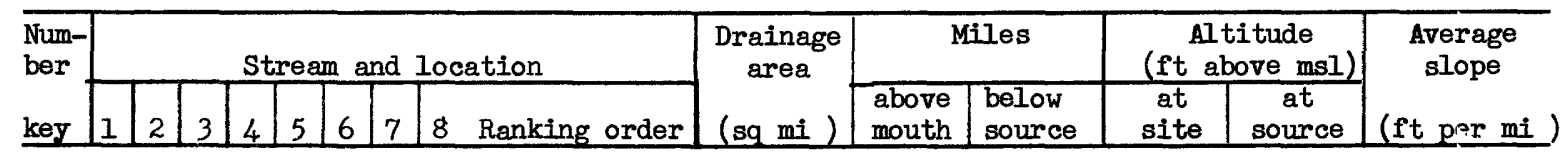

\begin{tabular}{|c|c|c|c|c|c|c|c|}
\hline 327 & $\begin{array}{l}\text { Newland Drain above unnamed } \\
\text { tributary, SWt sec. } 32, \mathrm{~T} .6 \mathrm{~N} . \mathrm{y} \\
\text { R.13 E. }\end{array}$ & 2.51 & 4.8 & 2.7 & 782 & 798 & 5.9 \\
\hline 328 & $\begin{array}{l}\text { Unnamed tributary at mouth, } \\
\text { Sw } \frac{1}{4} \text { sec.32, T.6 N.,.R.13 E. }\end{array}$ & .99 & 0 & 2.2 & 782 & 814 & $11 . .5$ \\
\hline 329 & $\begin{array}{l}\text { Newlanci Drain at unnamed tributary, } \\
\text { SW } \frac{1}{4} \text { sec.32, T.6 N., R.13 E. }\end{array}$ & 3.50 & 4.8 & 2.7 & 782 & 798 & 5.9 \\
\hline زن & $\begin{array}{l}\text { livewland Drain above unnamed } \\
\text { trijutary, SE } \frac{1}{4} \text { sec.5, T.5 N., } \\
\text { R.13 E. }\end{array}$ & $4 \cdot 35$ & 3.7 & 3.8 & 775 & 798 & 6.1 \\
\hline 331 & $\begin{array}{l}\text { Unnamed tributary at mouth, } \\
\text { SE } \frac{1}{4} \text { sec.5, T.5 N., R.13 E. }\end{array}$ & 1.58 & 0 & 1.8 & 775 & 794 & 10.6 \\
\hline$\because: 2$ & $\begin{array}{l}\text { Newland Drain at unnamed tributary, } \\
\mathrm{SE}-\frac{1}{4} \text { sec.5, T.5 N., R.13 E. }\end{array}$ & 5.93 & 3.7 & 3.8 & $775^{\circ}$ & 798 & 6.1 \\
\hline$=3$ & $\begin{array}{l}\text { Newland Drain at mouth, SW/ } \\
\text { sec.19, T.5 N., R.13 E. }\end{array}$ & 9.38 & 0 & 7.5 & 745 & 798 & $7 \cdot 4$ \\
\hline 334 & $\begin{array}{l}\text { North Branch Clinton River at Newland } \\
\text { Drain, SW sec.19, T.5 N., } \\
\text { R.13 E. }\end{array}$ & 43.4 & 29.4 & 13.8 & 745 & 973 & 16.5 \\
\hline 335 & 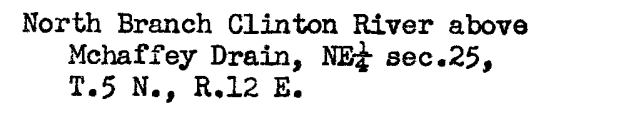 & 44.5 & 28.4 & 14.8 & 738 & 973 & 15.9 \\
\hline 336 & $\begin{array}{l}\text { Mchaffey Drain above Wilson Drain, } \\
\text { SE } \frac{1}{4} \text { sec.23, T.5 N., R.12 E. }\end{array}$ & 1.05 & 1.2 & 1.6 & 762 & 813 & $3 \cdots 9$ \\
\hline 337 & $\begin{array}{l}\text { Wilson Drain at mouth, SE- } \\
\text { sec.23, T.5 N., R.12 E. }\end{array}$ & .76 & 0 & 2.0 & 762 & 850 & $4+.0$ \\
\hline 338 & $\begin{array}{l}\text { Mchaffey Drain at Wilson Drain, } \\
\text { SE } \frac{1}{4} \text { sec.23, T.5 N., R.12 E. }\end{array}$ & 1.81 & 1.2 & 1.6 & 762 & 813 & $3-.9$ \\
\hline 339 & $\begin{array}{l}\text { Mchaffey Drain at Mack Road, } \\
\text { NWt sec.25, T.5 N., R.12 E. }\end{array}$ & 2.63 & .6 & 2.2 & 753 & 813 & 27.3 \\
\hline 340 & $\begin{array}{l}\text { Mchaffey Drain at mouth, } N E \frac{1}{4} \\
\text { sec.25, T.5 N., R.12 E. }\end{array}$ & 3.22 & 0 & 2.8 & 738 & 813 & 26.8 \\
\hline 347 & $\begin{array}{l}\text { North Branch Clinton River at Mchaffey } \\
\text { Drain, NBt sec.25, T.5 N., R.12 E. }\end{array}$ & 47.7 & 28.4 & 14.8 & 738 & 973 & 15.9 \\
\hline 342 & $\begin{array}{l}\text { North Branch Clinton River above } \\
\text { Farley Drain, SW } \frac{1}{4} \text { sec. } 30 \text {, T. } 5 \text { N., } \\
\text { R.13 E. }\end{array}$ & 49.0 & 27.4 & 15.8 & 732 & 973 & 15.3 \\
\hline 343 & $\begin{array}{l}\text { Farley Drain at mouth, SW- } \\
\text { sec. } 30 \text {, T. } 5 \text { N., R. } 13 \text { E. }\end{array}$ & .78 & 0 & 1.9 & 732 & 773 & 21.6 \\
\hline 344 & $\begin{array}{l}\text { North Branch Clinton River at Farley } \\
\text { Drain, SW sec.30, T.5 N., R.13 E. }\end{array}$ & 49.7 & 27.4 & 15.8 & 732 & 973 & 15.3 \\
\hline
\end{tabular}


PHYSIOGRAPHIC DATA FOR SELECTED LOCATIONS IN THE CLINTON RIVER BASIN-CONTINUED

\begin{tabular}{|c|c|c|c|c|c|c|c|c|c|c|c|c|c|c|c|}
\hline \multirow{2}{*}{$\begin{array}{l}\text { Num- } \\
\text { ber } \\
\text { key }\end{array}$} & \multicolumn{9}{|c|}{ Stream and location } & \multirow{2}{*}{$\begin{array}{c}\text { Drainage } \\
\text { area } \\
\text { (sq mi ) }\end{array}$} & \multicolumn{2}{|c|}{ Miles } & \multicolumn{2}{|c|}{$\begin{array}{c}\text { Altitude } \\
\text { (ft above msl) }\end{array}$} & \multirow{2}{*}{$\begin{array}{c}\text { Average } \\
\text { slope }\end{array}$} \\
\hline & 1 & 2 & 3 & 4 & 5 & 6 & 7 & 8 & Ranking order & & $\begin{array}{l}\text { above } \\
\text { mouth }\end{array}$ & $\begin{array}{l}\text { below } \\
\text { source }\end{array}$ & $\begin{array}{l}\text { at } \\
\text { site }\end{array}$ & $\begin{array}{c}\text { at } \\
\text { source }\end{array}$ & \\
\hline
\end{tabular}

345

North Branch Clinton River at 33-mile

Road (gage 04-16405), NW/ sec.31,

T. 5 N., R.13 E.

North Branch Clinton River above

East Pond Creek, SE $\frac{1}{4}$ sec.3l,

T. 5 N., R.13 E.

27.

15.8

50.6

26.

16.9

717

973

15.1

East Pond Creek at Secord Lake Road, SW $\frac{1}{4}$ sec.I, T. 5 N., R.11 E.

8.18

12.9

3.

$9.49 \quad 11.4$

5.

SW $\frac{1}{4}$ sec.7, T. 5 N., R. 12 E.

349

East Pond Creek at Schoof Road,

11.6

SW $\frac{1}{4}$ sec.17, T. 5 N., R.12 E.

350

351

East Pond Creek at Schooley

Road, SW $\frac{1}{4}$ sec.29, T. 5 N.,

R.12 E.

$\begin{array}{llllll}14.2 & 7.8 & 8.9 & 858 & 1000 & 16.0\end{array}$

Fast Pond Creek at Fast Mill Iake

6.

outlet, SW $\frac{1}{4}$ sec. 33 , T. 5 N.,

R.12 E.

352

East Pond Creek above Hidden Lake 19.0

outlet, NW $\frac{1}{4}$ sec.27, T. 5 N., T. 12 E.

353

Hidden Lake outlet at mouth,

2.280

$\begin{array}{llll}4.2 & 12.5 & 784 & 1000\end{array}$

17.3

NW $\frac{1}{4}$ sec. 27, T. 5 N., R.12 E.

354

355

East Pond Creek at Hidden Lake

outlet, NW $\frac{1}{4}$ sec. 27 , T. 5 N.,

R.12 E.

$\begin{array}{llllll}21.3 & 4.2 & 12.5 & 784 & 1000 & 17.3\end{array}$

East Pond Creek at Highway 53

(gage 04-164l), SE-1 sec.27, T. 5 N.,

21.8

3.6

13.1

775

1000

17. ล.

R.12 E.

356

East Pond Creek at Powell Road,

24.5

.

$\mathrm{SE} \frac{1}{4}$ sec. 36, T. 5 N., R. 12 E.

357

East Pond Creek at mouth, $\mathrm{SE}_{\frac{1}{4}}^{\frac{1}{4}}$

sec. 31, T. 5 N., R. 13 E.

26.

26.3

0

76.

North Branch Clinton River at East

Pond Creek, $\mathrm{SE}_{\frac{1}{4}}^{\frac{1}{4}}$ sec. 31, T. 5

N., R.13 E.

North Branch Clinton River above

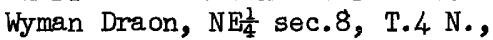
R.13 E.

360

Wyman Drain at mouth, $\mathrm{NE}_{\frac{1}{4}}$ sec.8, T. 4 N., R. 13 E.

361 North Branch Clinton River at Wyman Drain, $\mathrm{NE}_{4}^{\frac{1}{4}}$ sec.8, T.4 N., R.13 E.

$\begin{array}{llllll}77.9 & 24.0 & 19.2 & 687 & 973 & 14 . \mathrm{C}\end{array}$

$\begin{array}{llllll}1.43 & 0 & 1.6 & 687 & 735 & 30.0\end{array}$

$\begin{array}{llllll}79.3 & 24.0 & 19.2 & 687 & 973 & 14.9\end{array}$ 
PHYSIOGRAPHIC DATA FOR SELECTED LOCATIONS IN THE CLINTON RIVER BASIN-CONTINUED

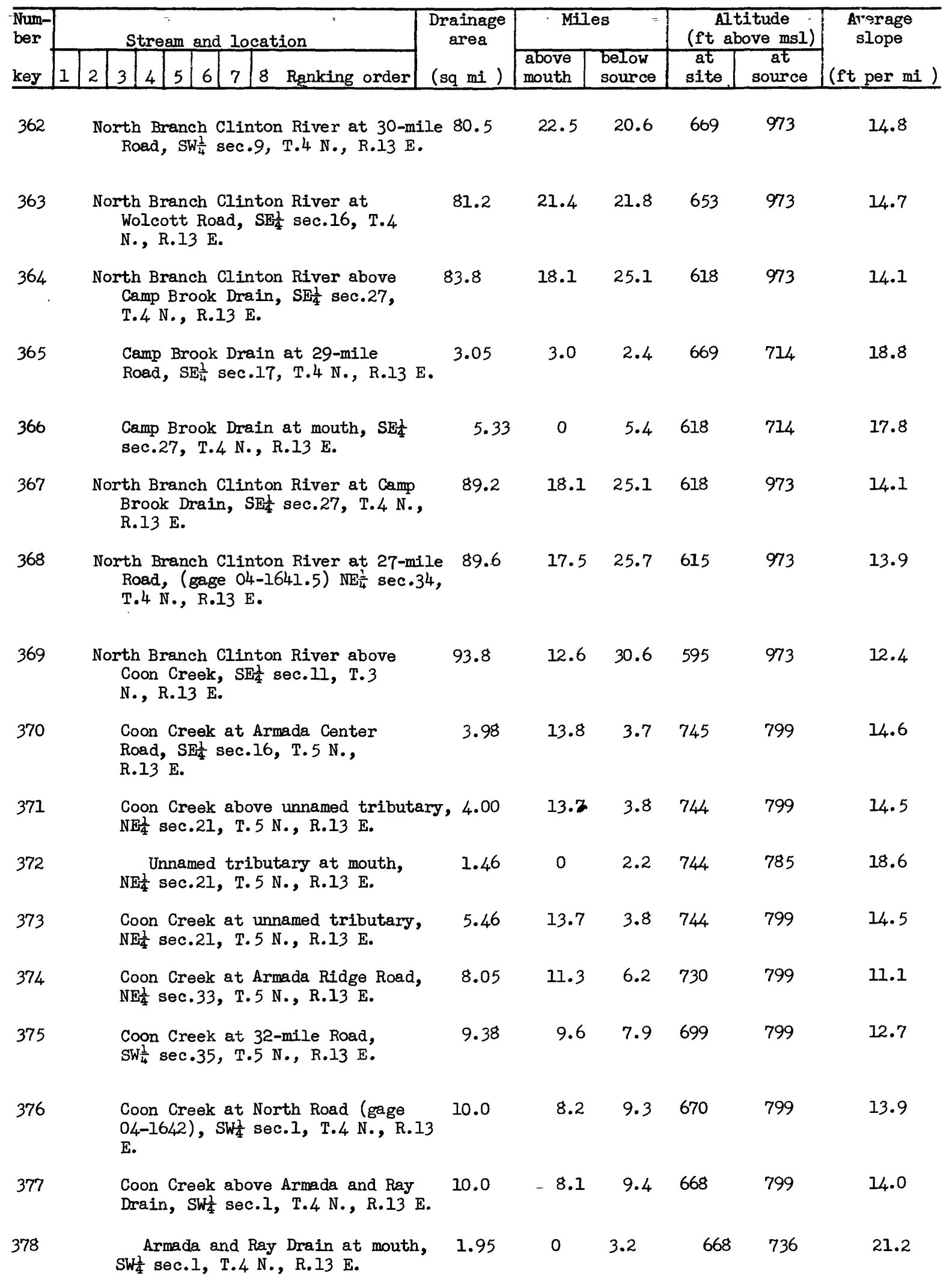


PHYSIOGRAPHIC DATA FOR SELECTED LOCATIONS IN THE CLINTON RIVER BASIN-CONTINUED

\begin{tabular}{|c|c|c|c|c|c|c|c|c|}
\hline \multirow{2}{*}{$\begin{array}{l}\text { Num- } \\
\text { ber } \\
\text { key }\end{array}$} & \multicolumn{2}{|r|}{ Stream and location } & \multirow{2}{*}{$\begin{array}{l}\text { Drainage } \\
\text { area } \\
(\mathrm{sg} \mathrm{mi}) \\
\end{array}$} & \multicolumn{2}{|c|}{ Miles } & \multicolumn{2}{|c|}{$\begin{array}{c}\text { Altitude } \\
\text { (ft above msl) }\end{array}$} & \multirow{2}{*}{$\begin{array}{c}\begin{array}{c}\text { Average } \\
\text { slope }\end{array} \\
\text { (ft per mi) }\end{array}$} \\
\hline & 12 & \begin{tabular}{l|l|l|l|l|l|l|}
3 & 4 & 5 & 6 & 7 & 8 & Ranking order \\
\end{tabular} & & $\begin{array}{l}\text { above } \\
\text { mouth }\end{array}$ & $\begin{array}{l}\text { below } \\
\text { source }\end{array}$ & $\begin{array}{c}\text { at } \\
\text { site }\end{array}$ & $\begin{array}{c}\text { at } \\
\text { source }\end{array}$ & \\
\hline 379 & & $\begin{array}{l}\text { Coon Creek at Armada and Ray } \\
\text { Drain, SW } \frac{1}{4} \text { sec.1, T. } 4 \text { N., } \\
\text { R.13 E. }\end{array}$ & 12.0 & 8.1 & 9.4 & 668 & 799 & 14.0 \\
\hline 380 & & $\begin{array}{l}\text { Coon Creek at North Road, SW } \frac{1}{4} \\
\text { sec.25, T. } 4 \text { N., R.13 E. }\end{array}$ & 15.2 & 3.7 & 13.8 & 614 & 799 & 13.4 \\
\hline 381 & & $\begin{array}{l}\text { Coon Creek above Tupper Brook, } \\
\text { NE⿺ sec. } 35 \text {, T. } 4 \text { N., R. } 13 \text { E. }\end{array}$ & 15.5 & 2.8 & 14.7 & 605 & 799 & 13.2 \\
\hline 382 & & $\begin{array}{l}\text { Tupper Brook at Wolcott Road, } \\
\text { SE } \frac{1}{4} \text { sec. } 32 \text {, T. } 5 \text { N., R. } 13 \text { E. }\end{array}$ & 2.13 & 8.8 & 2.0 & 737 & 769 & 16.0 \\
\hline 383 & & $\begin{array}{l}\text { Tupper Brook at } 31 \text {-mile Road, } \\
\mathrm{SE}_{\frac{1}{4}}^{\frac{1}{4}} \text { sec.2, T.4 N., R.13 E. }\end{array}$ & 4.21 & 5.3 & 5.5 & 674 & 769 & 17.3 \\
\hline 384 & & $\begin{array}{l}\text { Tupper Brook above Priest } \\
\text { Drain, SW } \frac{2}{4} \text { sec.14, T. } 4 \text { N., } \\
\text { R.13 E. }\end{array}$ & 5.88 & 3.1 & 7.7 & 639 & 769 & 16.9 \\
\hline 385 & & $\begin{array}{l}\text { Priest Drain at mouth, } \\
\text { SW }\end{array}$ & 2.68 & 0 & 3.8 & 639 & 724 & 2.2 .4 \\
\hline 386 & & $\begin{array}{l}\text { Tupper Brook at Priest Drain, } \\
\text { SW }\end{array}$ & 8.56 & 3.1 & 7.7 & 639 & 769 &. .6 .9 \\
\hline 387 & & 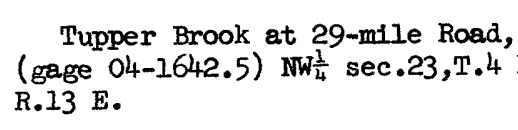 & N., 8.62 & 3.0 & 7.8 & 637 & 769 & $=.6 .9$ \\
\hline 388 & & $\begin{array}{l}\text { Tupper Brook at mouth, } \mathrm{NE}_{4} \\
\text { sec.35, T. } 4 \text { N., R.13 E. }\end{array}$ & 10.6 & 0 & 10.8 & 605 & 769 & $=.5 .2$ \\
\hline 389 & & 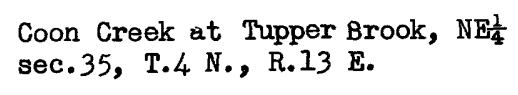 & 26.1 & 2.8 & 14.7 & 605 & 799 & 1.3 .2 \\
\hline 390 & & $\begin{array}{l}\text { Coon Creek above East Branch } \\
\text { Coon Creek, SE sec. } 35 \text {, T. } 4 \text { N., } \\
\text { R.13 E. }\end{array}$ & 26.4 & 2.3 & 15.2 & 603 & 799 & 12.9 \\
\hline 39 & & $\begin{array}{l}\text { East Branch Coon Creek above } \\
\text { unnamed tributary, SW } \frac{1}{4} \text { sec.22, } \\
\text { T.6 N., R.13 E. }\end{array}$ & 1.97 & 21.2 & 2.0 & 789 & 813 & 32.0 \\
\hline$\therefore$ & & $\begin{array}{l}\text { Unnamed tributary at } \\
\text { nouih, SW } \frac{1}{4} \text { sec.22, T.6 N., } \\
\text { R.13 E. }\end{array}$ & 1.55 & 0 & 1.8 & 789 & 795 & 3.3 \\
\hline 3,3 & & $\begin{array}{l}\text { East Branch Coon Creek at } \\
\text { unnamed tributary, SW } \frac{1}{4} \text { sec.22, } \\
\text { T.6 N., R.13 E. }\end{array}$ & 3.52 & 21.2 & 2.0 & 789 & 813 & 32.0 \\
\hline 304 & & $\begin{array}{l}\quad \text { East Branch Coon Creek at } \\
\text { Pratt Road, NE } \frac{1}{4} \text { sec.2, T.5 N., } \\
\text { R.13 E. }\end{array}$ & 8.34 & 17.2 & 6.0 & 772 & 813 & 6.8 \\
\hline $3 \pm 5$ & & 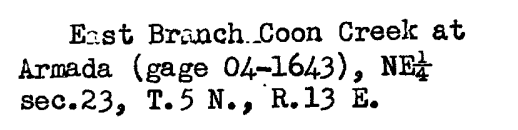 & 13.0 & 13.3 & 9.9 & 735 & 813 & 7.9 \\
\hline
\end{tabular}


PHYSIOGRAPHIC DATA FOR SELECTED LOCATIONS IN THE CLINTON RIVER BASIN--CONTINUED

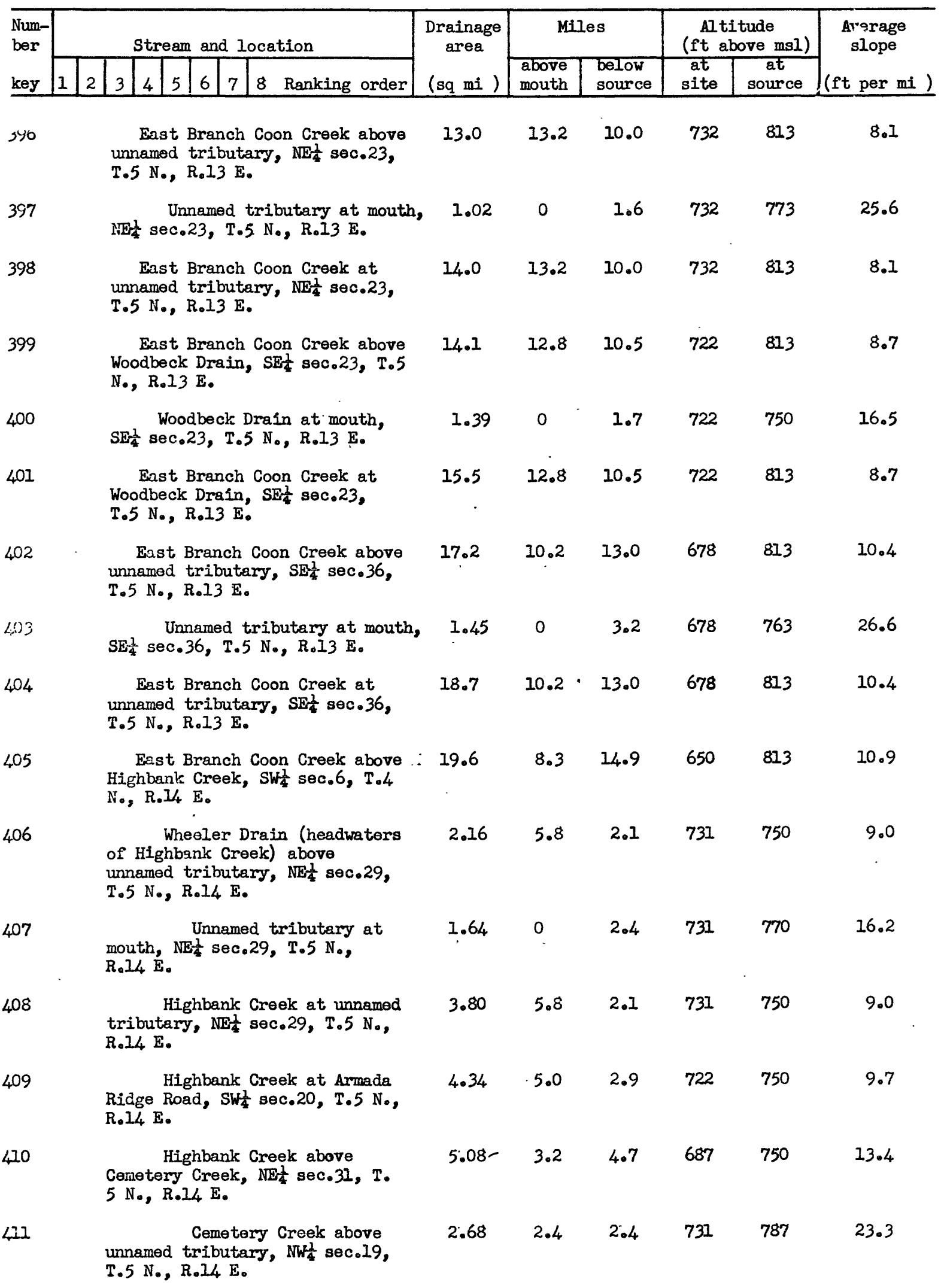


PHYSIOGRAPHIC DATA FOR SELECTED LOCATIONS IN THE CLINTON RIVER BASIN--CONTINUED

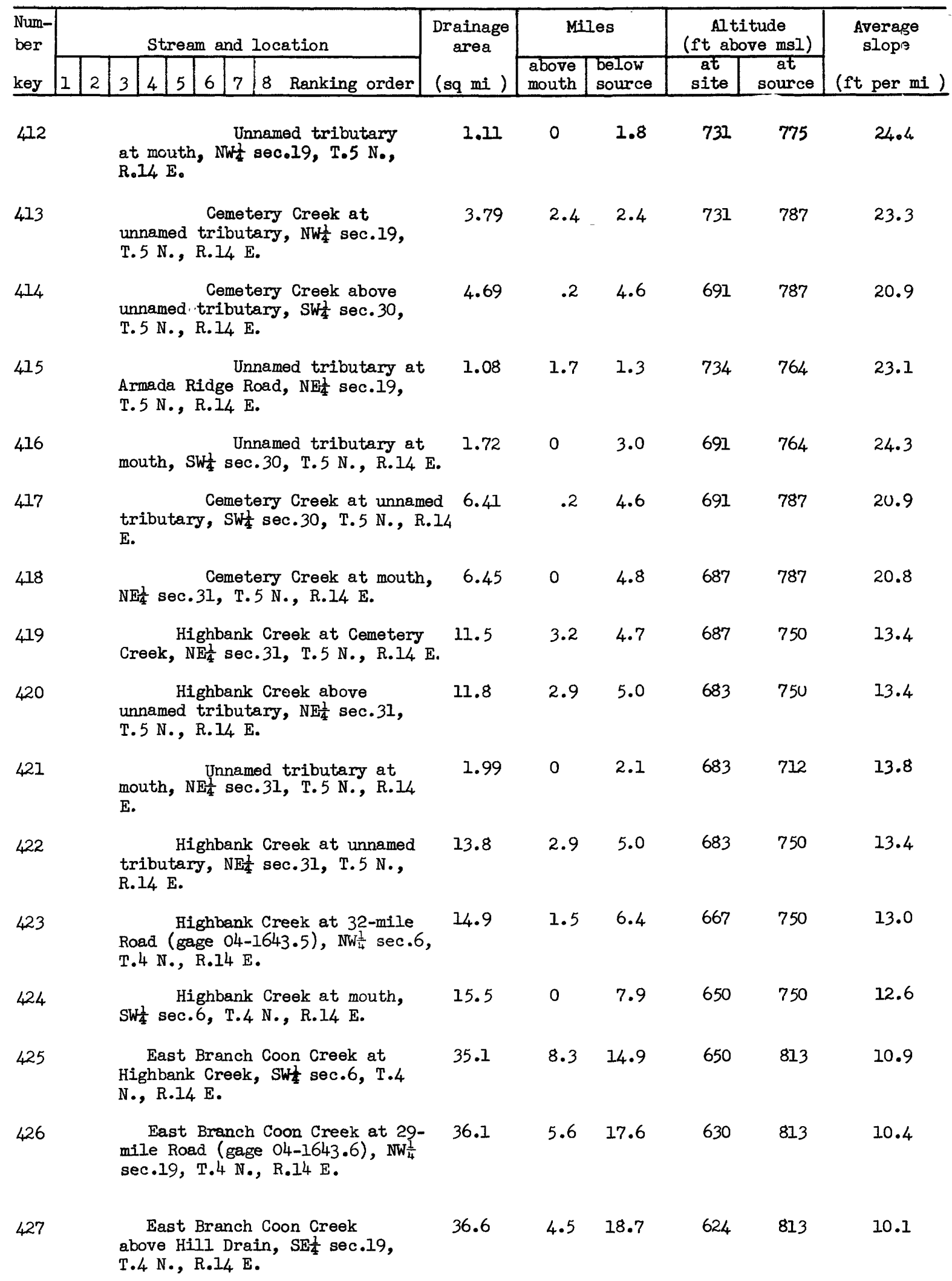


PHYSIOGRAPHIC DATA FOR SELECTED LOCATIONS IN THE CLINTON RIVER BASIN-CONTINUED

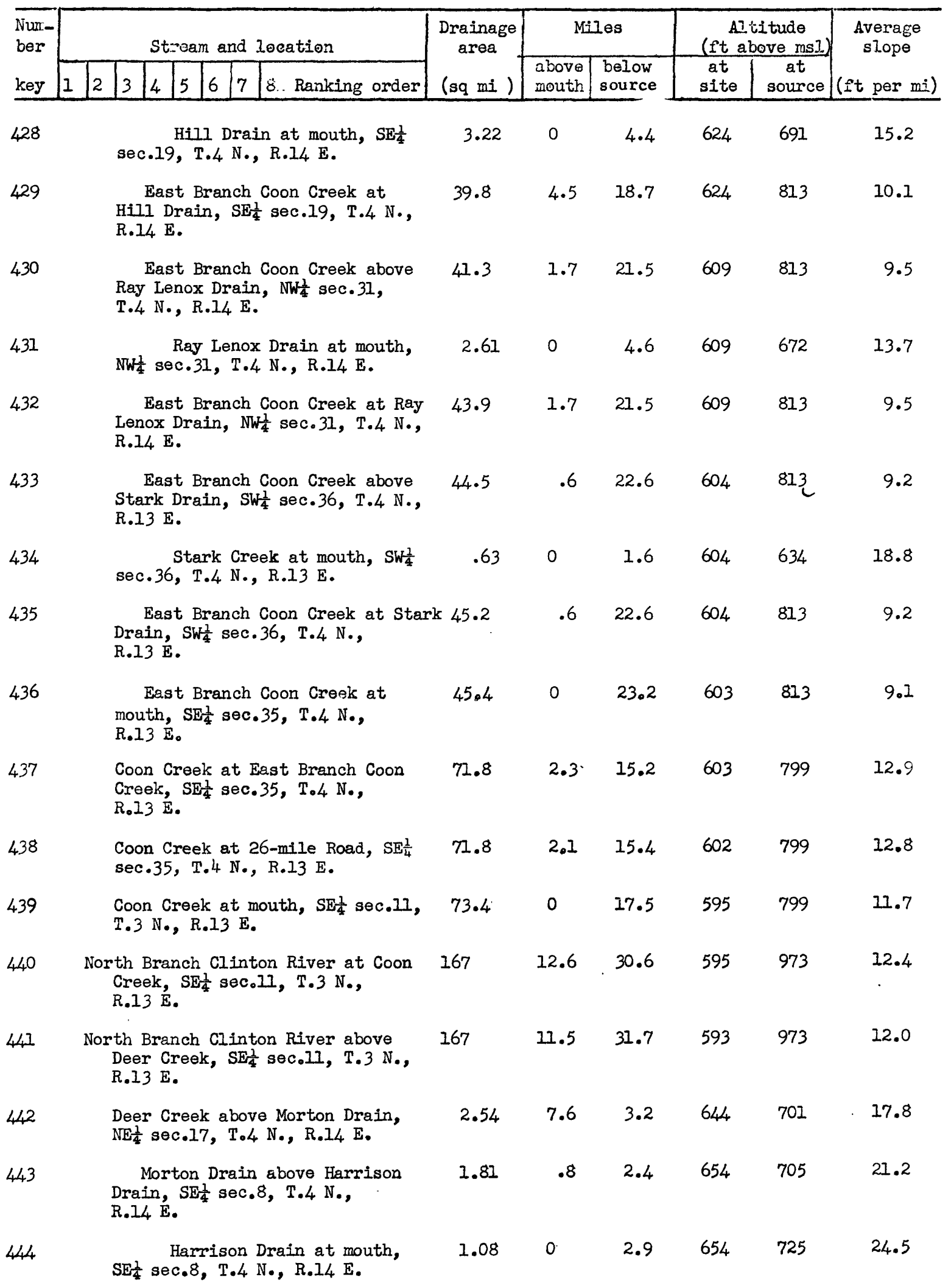


PHYSIOGRAPHIC DATA FOR SETECTED LOCATIONS IN THE CLINTON RIVER BASIN-CONTINUED

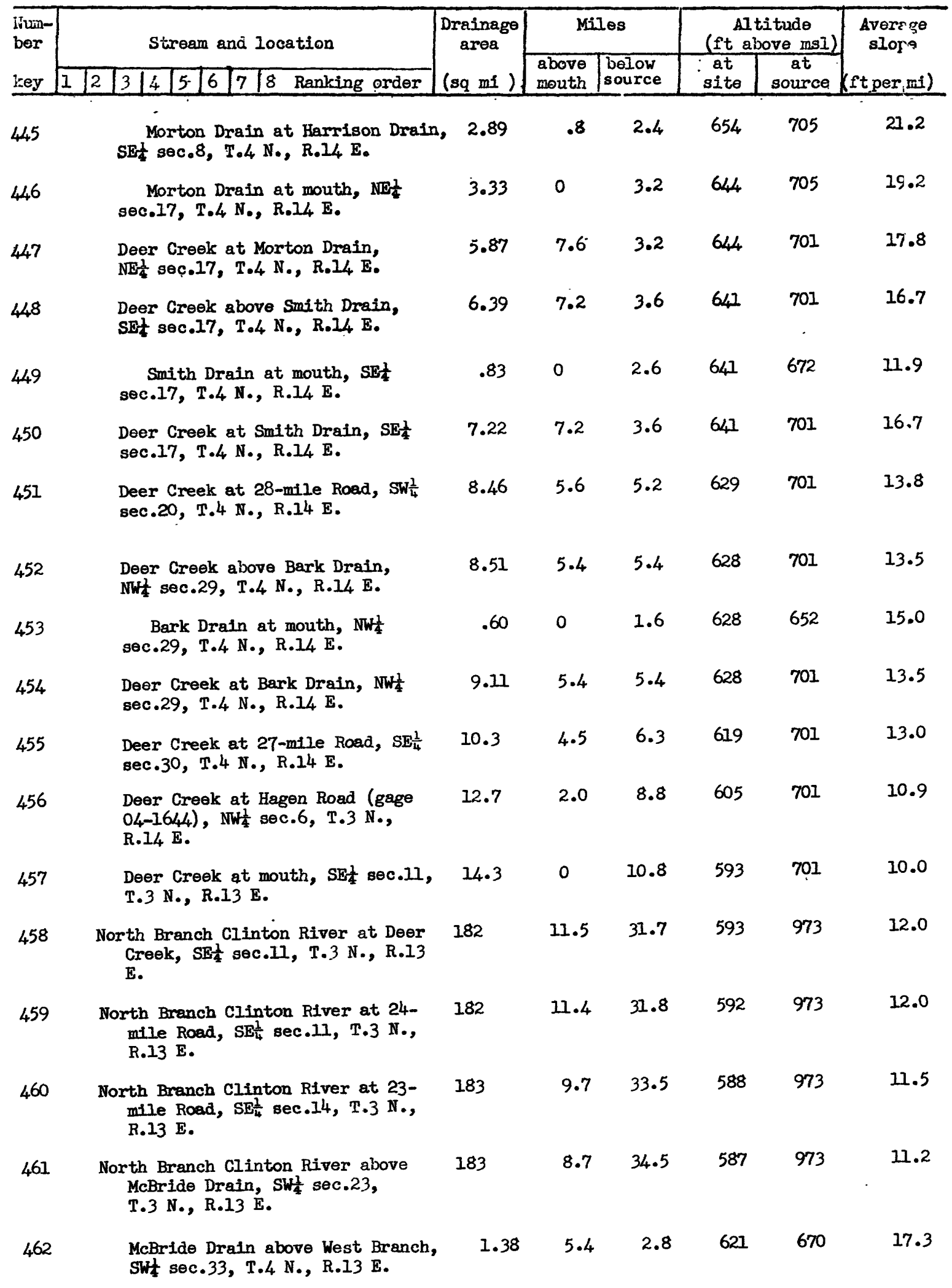


PHYSIOGRAPHIC DATA FOR SELECTED LOCATIONS IN THE CLTNTON RIVER BASIN-CONTTNUED

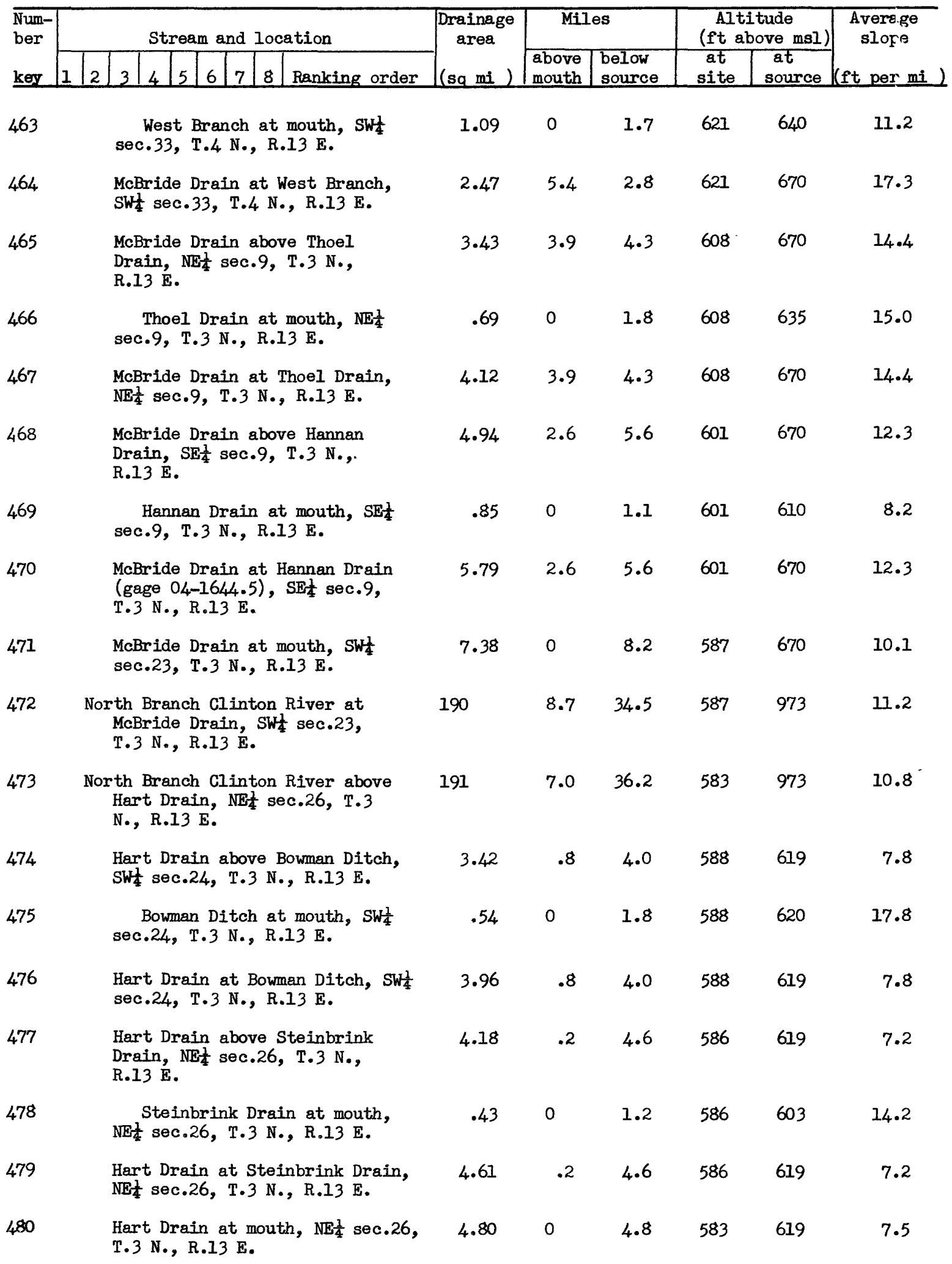


PHYSIOGRAPHIC DATA FOR SELECIED LOCATIONS IN THE CLINTON RIVER BASIN--CONTINUED

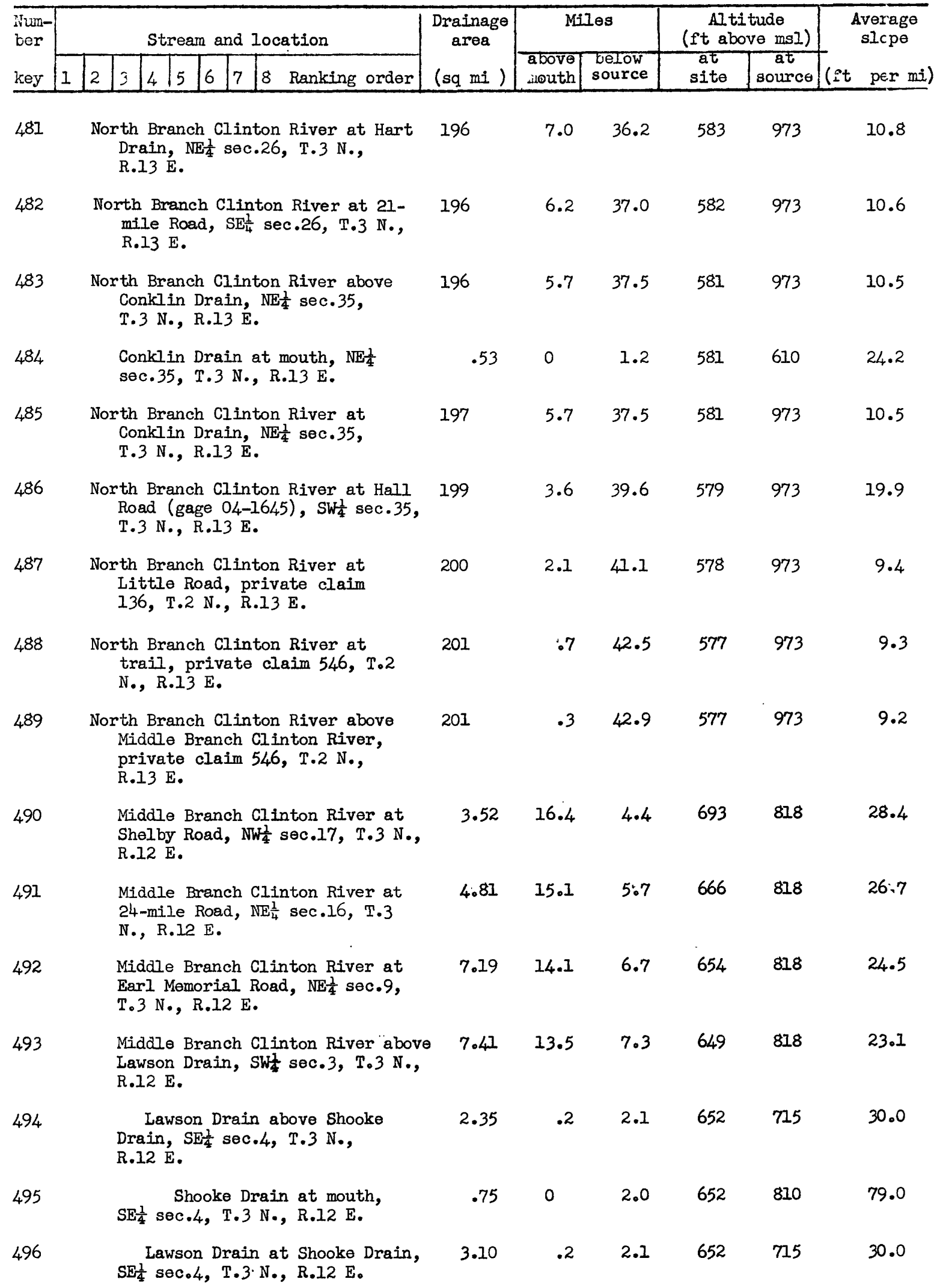


PHYSIOGRAPHIC DATA FOR SELECTED LOGATIONS IN THE CLTNTON RIVER BASIN--CONTTNUED

\begin{tabular}{|c|c|c|c|c|c|c|c|c|c|c|c|c|c|c|}
\hline \multirow{2}{*}{$\begin{array}{l}\text { Num- } \\
\text { ber } \\
\text { key }\end{array}$} & \multicolumn{8}{|c|}{ Stream and location } & \multirow{2}{*}{$\begin{array}{l}\text { Drainage } \\
\text { area } \\
\text { (sq mi ) }\end{array}$} & \multicolumn{2}{|c|}{ Miles } & \multicolumn{2}{|c|}{$\begin{array}{c}\text { Altitude } \\
\text { (ft above msl) }\end{array}$} & \multirow{2}{*}{$\begin{array}{c}\text { Av arage } \\
\text { stope } \\
\text { (ft per mi }\end{array}$} \\
\hline & 12 & 3 & 4 & 5 & 6 & 7 & 8 & Ranking order & & $\begin{array}{l}\text { above } \\
\text { mouth }\end{array}$ & $\begin{array}{l}\text { below } \\
\text { source }\end{array}$ & $\begin{array}{c}a t \\
\text { site }\end{array}$ & $\begin{array}{c}\text { at } \\
\text { source }\end{array}$ & \\
\hline
\end{tabular}

497

498

501

502

504

505

506

507

508

509

510

511

512

513

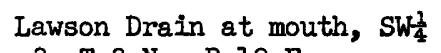

Middle Branch Clinton River at Lawson Ditch, SWt sec.3, T.3 N., R.12 E.

Middle Branch Clinton River above Cannon Drain, SW $\frac{1}{4}$ sec.3, T.3 N., R.12 E.

\subsection{4}

10.6

13.5

10.8

13.2

.830

1.2

645

698

715

28.7 sec.3, T.3 N., R.12 E.

Middle Branch Clinton River at Cannon Drain, SW $\frac{1}{4}$ sec.3, T. 3 N., R.12 E.

11

11.7

Middle Branch Clinton River above Disco Drain, SE $\frac{1}{4}$ sec.3, T. 3 N., R.12 E.

Disco Drain at mouth, $\mathrm{SE} \frac{-1}{4}$ sec.3, T. 3 N., R.12 E.

$\begin{array}{llllll}.43 & 0 & 1.5 & 643 & 660 & 11.3\end{array}$

12.

Middle Branch Clinton River at Disco Drain, SE $\frac{1}{4}$ sec.3, T. 3 N., R.12 E.

Middle Branch Clinton River above Hawald Drain, SE $\frac{1}{4}$ sec.

12. 3., T.3 N., R.12 E.

Hawald Drain at mouth, SE $\frac{1}{4}$ sec.3, T.3 N., R.I2 E.

Middle Branch Clinton River at Hawald Drain, SE $\frac{1}{4}$ sec.3, T.3 N., R.12 E.

Middle Branch Clinton River above Keller Drain, SW $\frac{7}{4}$ sec.2, T.3 N., R.12 E.

$13.0 \quad 12.4$

8.4

1.2

643

818

14.6

13.0

12.7

8.1

643

818

21.6

Keller Drain above Yates Drain, 2.36

1.8 SW $\frac{1}{4}$ sec.26, T.4 N., R.12 E.

$$
\begin{aligned}
& \text { Yates Drain at mouth, SWt } \\
& \text { sec.26, T.4 N., R.12 E. }
\end{aligned}
$$

Keller Drain at Yates Drain, SWt sec.26, T.4 N., R.12 E.

$\begin{array}{llllll}3.34 & 1.8 & 2.6 & 672 & 840 & 64.6\end{array}$

Keller Drain above unnamed tributary, NW $\frac{1}{4}$ sec. $35, \mathrm{~T} .4 \mathrm{~N}$. , R.12 E.

$$
\begin{aligned}
& \text { Unnamed tributary at } \\
& \text { mouth, NW } \frac{1}{4} \text { sec.35, T.4 N., R. } \\
& 12 \mathrm{E} .
\end{aligned}
$$

$\begin{array}{llllll}3.36 & 1.7 & 2.7 & 671 & 840 & 62.6\end{array}$

$\begin{array}{llllll}1.42 & 0 & 2.6 & 671 & 838 & 64.2\end{array}$


PHYSIOGRAPHIC DATA FOR SETECTED LOCATIONS IN THE CLINTON RIVER BASIN-CONTINUED

\begin{tabular}{|c|c|c|c|c|c|c|c|c|c|c|c|c|c|c|c|}
\hline $2 m-1$ & \multicolumn{9}{|c|}{ Stream and location } & \multirow{2}{*}{$\begin{array}{c}\text { Drainage } \\
\text { area } \\
(\mathrm{sq} \mathrm{mi})\end{array} \mid$} & \multicolumn{2}{|c|}{ Miles } & \multicolumn{2}{|c|}{$\begin{array}{c}\text { Altitude } \\
\text { (ft s.bove } \mathrm{ms} \text { ) }\end{array}$} & \multirow{2}{*}{$\begin{array}{c}\text { Avejage } \\
\text { slope } \\
\text { (it per mi) }\end{array}$} \\
\hline & 12 & 2 & 3 & 4 & 5 & 6 & 7 & 0 & Ranking order & & $\begin{array}{l}\text { above } \\
\text { mouth }\end{array}$ & $\begin{array}{l}\text { below } \\
\text { source }\end{array}$ & $\begin{array}{l}\text { at } \\
\text { site }\end{array}$ & $\begin{array}{c}a t \\
\text { source }\end{array}$ & \\
\hline
\end{tabular}

514

515

516

517

518

519

520

521

522

523

524

525

526

527

528

529
Keller Drain at unnamed tributary, NW $\frac{1}{4}$ sec.35, T.4 N., R.12 E.

Keller Drain above unnamed tributary, SE $\frac{1}{4}$ sec.34, T.4 N., R.12 E. Unnamed tributary at
mouth, SEt sec.34, T.4 N., R.12 E

Keller Drain a.t unnamed tributary, SE $\frac{1}{4}$ sec. 34, T. 4 N., R.12 E.

Keller Drain above unnamed

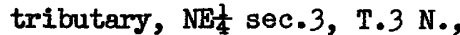
R.12 E. Unnamed tributary at
NEt sec.3, T.3 N., R.12 E.

Keller Drain at unnamed tributary, NEt sec.3, T.3 N., R.12 E.

Keller Drain at mouth, $\mathrm{SW}_{4}^{\frac{1}{4}}$ sec.2, T.3 N., R.12 E.

Middle Branch Clinton River at

Keller Drain, SW $\frac{1}{4}$ sec.2, T.3 N., R.12 E.

Middle Branch Clinton River at Schoenherr Road (gage 04-1646), SEl sec.2, T.3 N., R.12 E.

Middle Branch Clinton River above Healy Drain, NWt $\frac{1}{4}$ sec.6, T. 3 N., R.13 E.

1

$\begin{array}{llllll}4.78 & 1.7 & 2.7 & 671 & 840 & 6 ? .6\end{array}$

5.26

.8

3.6

654

840

51.7

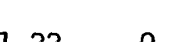

0

1.8

654

787

73.9

$\begin{array}{llllll}6.48 & .8 & 3.6 & 654 & 840 & 51.7\end{array}$

$\begin{array}{llllll}6.59 & .6 & 3.8 & 649 & 840 & 57.3\end{array}$

(1)

onth

,

1.15

0

7.74

$2.4 \quad 649 \quad 810$

67.1

7.74

.6

3.8

649

840

57.3

8.420

$21.4 \quad 12.4$

4.4

639

840

45.7

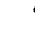

22

22.2

11.4

8.4

639

818

21.3

Healy Drain at Jewell Road, NWt sec.11, T.4 N., R.12 E.

$\begin{array}{llllll}23.6 & 9.8 & 11.0 & 614 & 818 & 18.5\end{array}$

2.28

1.9

780

849

$3 \cdot 3$

3.22

5.8

4.0

707

849

$3 \div .5$

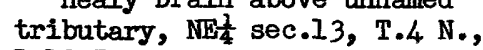
R.12 E.

1.18

0

$2.7 \quad 707$

847

51.9 NEt sec.13, T.4 N., R.12 E.

$\begin{array}{llllll}4.40 & 5.8 & 4.0 & 707 & 849 & 3 \% .5\end{array}$

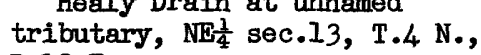
R.12 E.

4.40

9.65

.6

9.2

622

849

24.7 $\mathrm{SE}_{\frac{1}{4}} \mathrm{sec} \cdot 31$, T.4 N., R.13 E.

Healy Drain above Price Brook, NWt sec.6, T.3 N.,

9.84 .1

9.7

614

849

24.2 R.13 E. 
PHYSIOGRAPHIC DATA FOR SELECTED LOCATIONS IN THE CLTNTON RIVER BASIN--CONTINUTD

\begin{tabular}{|c|c|c|c|c|c|c|c|c|c|c|c|c|c|c|c|}
\hline $\begin{array}{l}\text { Num- } \\
\text { ber }\end{array}$ & \multicolumn{9}{|c|}{ Stream and location } & \multirow{2}{*}{$\begin{array}{l}\text { Drainage } \\
\text { area } \\
\text { (sa mi ) }\end{array}$} & \multicolumn{2}{|c|}{ Miles } & \multicolumn{2}{|c|}{$\begin{array}{c}\text { Altitude } \\
\text { (ft above msl) }\end{array}$} & \multirow{2}{*}{$\begin{array}{c}\text { Avirage } \\
\text { slope } \\
\text { (ft per mi ) }\end{array}$} \\
\hline key & 1 & 2 & 3 & 4 & 5 & 6 & 7 & 8 & Ranking order & & $\begin{array}{l}\text { above } \\
\text { mouth }\end{array}$ & $\begin{array}{l}\text { below } \\
\text { source }\end{array}$ & $\begin{array}{l}\text { at } \\
\text { site }\end{array}$ & $\begin{array}{c}\text { at } \\
\text { source }\end{array}$ & \\
\hline
\end{tabular}

$\begin{array}{llllllll}531 & \text { Price Brook at mouth, } & 7.18 & 0 & 4.6 & 614 & 715 & 21.9\end{array}$ NWt sec.6, T.3 N., R.13 E.

532

Healy Drain at Price Brook,

17. NWt sec.36, T.3 N., R.13 E.

533

Healy Drain at mouth, Nwt sec.6, T.3 N., R.13 E.

534

Middle Branch Clinton River at Healy Drain, NW $\frac{1}{4}$ sec.6, T. 3 N., R.13 E. 1648), SW sec.5, T.3 N., R. $13 \mathrm{E}$.

Midale Branch Clinton River at 24-mile Rosd, $\mathrm{SE}_{\frac{1}{4}} \mathrm{sec} .8$, T.3 N., R.13 E.

Middle Branch Clinton River above MaComb Ditch, NE $\frac{1}{4}$ sec.17, T.3 N.,

17.0

40.7

$\begin{array}{lllll}7 & 9.8 & 11.0 & 614 & 818\end{array}$
R.13 E.

$\begin{array}{llllll}41.0 & 9.1 & 11.7 & 608 & 818 & 17.9\end{array}$

$\begin{array}{llllll}41.9 & 7.4 & 13.4 & 601 & 818 & 16.2\end{array}$

$\begin{array}{llllll}42.1 & 7.2 & 13.6 & 599 & 818 & 16.1\end{array}$

MaComb Ditch at mouth, NEt sec.17, T.3 N., R.13 E.

$\begin{array}{llllll}1.34 & 0 & 1.6 & 599 & 625 & 16.2\end{array}$

Middle Branch Clinton River at MaComb Ditch, NEt sec.17, T.3 N., R.13 E.

43.4

$\begin{array}{llll}7.2 & 13.6 & 599 & 818\end{array}$

16.1

Midale Branch Clinton River at 23-mile Road, SE $\frac{1}{4}$ sec.17,

43.7

$6.4 \quad 14.4$

596

818

15.4 T.3 N., R.13 E.

541

Middle Branch Clinton River at 22-mile Road, SW $\frac{1}{4}$ sec.2l, T.3 N., R.13 E.

45.1

Middle Branch Clinton River at 2l-mile Road, SW $\frac{1}{4} \sec .28$,

46.0 T. 3 N., R.13 E.

543 Middle Branch Clinton River above Pingle Drain, NW $\frac{1}{4}$ sec. 33, T.3 N., R.13 E.

544

Pingle Drain at mouth, NW $\frac{1}{4}$ sec.33, T. 3 N., R.13 E.

Middle Branch Clinton River at Pingle Drain, NW $\frac{1}{4}$ sec.33, T.3

46.1

$3.8 \quad 17.0$

1.66

0

3.6

585

606

5.8 N., R.13 E.

47.

$\begin{array}{lllll}3.8 & 17.0 & 585 & 818 & 13.7\end{array}$

546

Middle Branch Clinton River above Heydenreich Drain, SW sec.33, T.3 N., R.13 E.

48.2

3.1

17.7

583

818

13.3

Heydenreich Drain at mouth, SW $\frac{1}{4}$ sec.33, T.3 N., R.13 E.

0

2.6

583

600

6.5 
PHYSIOGRAPHIC DATA FOR SELECTED LOCATIONS IN THE CLINTON RIVER BASIN--CONTINUED

\begin{tabular}{|c|c|c|c|c|c|c|c|c|c|c|c|c|c|c|}
\hline \multirow{2}{*}{$\begin{array}{l}\text { Num- } \\
\text { ber }\end{array}$} & \multicolumn{8}{|c|}{ Stream and location } & \multirow{2}{*}{$\begin{array}{l}\text { Drainage } \\
\text { area } \\
\text { (sq mi ) }\end{array}$} & \multicolumn{2}{|c|}{ Miles } & \multicolumn{2}{|c|}{$\begin{array}{c}\text { Altitude } \\
\text { (ft above msl) }\end{array}$} & \multirow{2}{*}{$\begin{array}{c}\text { Average } \\
\text { slope } \\
\text { (ft per mi ) }\end{array}$} \\
\hline & 1 & 2 & 3 & 4 & 5 & 6 & 7 & 8 Ranking order & & $\begin{array}{l}\text { above } \\
\text { mouth }\end{array}$ & $\begin{array}{l}\text { below } \\
\text { source }\end{array}$ & $\begin{array}{l}\text { at } \\
\text { site }\end{array}$ & $\begin{array}{l}\text { at } \\
\text { source }\end{array}$ & \\
\hline
\end{tabular}

548

549

550

Middle Branch Clinton River at Heydenreich Drain (at Hall Road, gage 04-1650), SW $\frac{1}{4}$ sec.33, T.3 N., R.13 E.

Middle Branch Clinton River above Gloede Ditch, $\mathrm{SE} \frac{1}{4}$ sec. 5 , T.2 N., R.13 E.

Harris Drain (headwaters of Gloede Ditch) above Longstaff Drain No.2, NW $\frac{1}{4}$ sec.19, T.3 N., R.13 E.

51 Longstaff Drain No. 2 at mouth, NW $\frac{1}{4}$ sec.19, T.3 N., R.13 E.

552

553

554

555

556

557

558

559

560

$50 ́ 1$

Gloede Ditch at Longstaff Drain No. 2, NW sec.19, T.3 N., R.13 E。

Gloede Ditch above Longstaff Drain, NW sec.19, T. $3^{\top} N_{0}$, R. $13 \mathrm{E}$ 。

Longstaff Drain at mouth NWt sec.19, T.3 N.; R.13 E.

Gloede Ditch at Longstaff Drain, NW sec.19, T.3 N., R.13 E。

Gloede Ditch above Lewis Drain, 7.2 SE $\frac{1}{4}$ sec. 31, T. 3 N., R.13 E.

$$
\text { Lewis Drain above Dunn }
$$
Drain, NWt sec.31, T.3 N., R.13 E.

48.8

$3.1 \quad 17.7$

583

818

13.3

49.0

$2.2 \quad 18.6$

2.77

$$
4.8 \quad 2.6
$$

604

643

15.0

0

$$
2.0
$$

604

639

15.5

3.53

4.

2.6

604

643

$15 . \mathrm{C}$

3.56

4.6

$4 \cdot 6$

2.8

602

643

14.6

2.27

0

3.4

602

659

16. E

40

4.6

2.8

602

643

14.6

2.0

5.4

589

643

10.0

.62

.8

2.1

594

609

7.1

Dunn Drain above Decker Drain, SE- sec.25, T.3 N.,

R.12 E.

Decker Drain above
Caflic Drain, SW sec.24, T.3

N., R.12 E.

$$
\begin{gathered}
\text { Caflic Drain at } \\
\text { mouth, SW } \frac{1}{4} \text { sec.24, T.3 N., R.12 E。 }
\end{gathered}
$$

Decker Drain at

Canlic Diain, SW $\frac{1}{4}$ sec.24, T.3

N., R.12 E.

5120

$\mathrm{SE} \frac{1}{4} \sec _{0} 25, \mathrm{~T} .3 \mathrm{~N} ., \mathrm{R}_{0} 12 \mathrm{E}$.

503 Dunn Drain at Decker

Drain, SE $\frac{1}{4}$ sec.25, T. 3 N., R. $12 \mathrm{E}$.
Decker Drain at mouth,

1.47

1.1

$2 \cdot 3$

670

656

20.0

.76

0

1.7

620

640

21.8

$2.23 \quad 1.1$

2.3

620

656

20.0

3.32

0

3.4

602

656

15.9

$4 \cdot 2$

1.2

2.0

602

636

17.0 
PHYSIOGRAPHIC DATA FOR SELECTED LOCATIONS IN THE CLINTON RIVER BASIN-CONTINUED

\begin{tabular}{|c|c|c|c|c|c|c|c|c|}
\hline \multirow{2}{*}{$\begin{array}{l}\text { Num- } \\
\text { ber } \\
\text { kery }\end{array}$} & \multicolumn{2}{|r|}{ Stream and location } & \multirow{2}{*}{$\begin{array}{l}\text { Drainage } \\
\text { area } \\
\text { (sq.mi) }\end{array}$} & \multicolumn{2}{|c|}{ Miles } & \multicolumn{2}{|c|}{$\begin{array}{c}\text { Altitude } \\
\text { (ft above msl) }\end{array}$} & \multirow{2}{*}{$\begin{array}{l}\text { Avejage } \\
\text { S.ope } \\
\text { (ft per mi) }\end{array}$} \\
\hline & $1|2|$ & \begin{tabular}{l|l|l|l|l|l}
3 & 4 & 5 & 6 & 7 & 8 Ranking order \\
\end{tabular} & & $\begin{array}{l}\text { above } \\
\text { mouth }\end{array}$ & $\begin{array}{l}\text { below } \\
\text { source }\end{array}$ & $\begin{array}{c}\text { at } \\
\text { site }\end{array}$ & $\begin{array}{c}\text { at } \\
\text { source }\end{array}$ & \\
\hline $50 \dot{4}$ & & $\begin{array}{l}\text { Dunn Drain above Wilcox } \\
\text { Drain, SE } \frac{1}{4} \text { sec.25, T.3 N., R.12 E. }\end{array}$ & 4.29 & .7 & 2.5 & 598 & 636 & 15.2 \\
\hline 565 & & $\begin{array}{l}\text { Wilcox Drain at mouth, } \\
\text { SE } \frac{1}{4} \text { sec.25, T.3 N., R.12 E. }\end{array}$ & .67 & 0 & 2.6 & 600 & 638 & 14.6 \\
\hline 506 & & $\begin{array}{l}\text { Dunn Drain at Wiicox } \\
\text { Drain, SE } \frac{1}{4} \text { sec.25, T.3 N., } \\
\text { R.12 E. }\end{array}$ & 4.90 & .7 & 2.5 & 598 & 636 & 15.2 \\
\hline 567 & & $\begin{array}{l}\text { Dunn Drain above } \\
\text { Shoemaker Drain, NE } \frac{1}{4} \text { sec.36, } \\
\text { T.3 N., R.12 E. }\end{array}$ & 4.98 & .5 & 2.7 & 596 & 636 & $u_{4.8}$ \\
\hline 568 & & $\begin{array}{l}\text { Shoemaker Drain at } \\
\text { mouth, NEt sec.36, T.3 N., } \\
\text { R.12 E. }\end{array}$ & .81 & 0 & 1.4 & 596 & 619 & 16.4 \\
\hline 569 & & 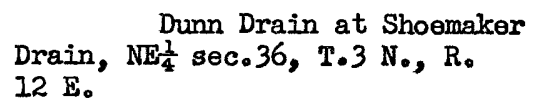 & 5.79 & .5 & 2.7 & 596 & 636 & 14.8 \\
\hline 570 & & 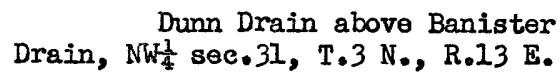 & 5.84 & .3 & 2.9 & 594 & 636 & 14.5 \\
\hline 571 & & $\begin{array}{c}\text { Banister Drain at } \\
\text { mouth, NW } \frac{1}{4} \text { sec.31, T.3 N., R.13 E. }\end{array}$ & 1.62 & 0 & 3.2 & 594 & 637 & 13.4 \\
\hline 572 & & 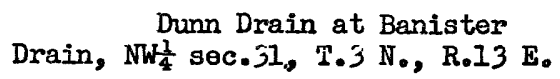 & 7.46 & .3 & 2.9 & 594 & 636 & 14.5 \\
\hline 573 & & $\begin{array}{l}\text { Dunn Drain at mouth, } \\
\text { NW } \frac{1}{4} \text { sec. } 31, \text { T. } 3 \text { N., R.I3 E. }\end{array}$ & 7.60 & 0 & 3.2 & 594 & 636 & 13.1 \\
\hline 574 & & $\begin{array}{l}\text { Lewis Drain at Dunn Drain, } \\
\text { NW } \frac{1}{4} \text { sec. } 31 \text {, T. } 3 \text { N., R.13 E. }\end{array}$ & 8.22 & .8 & 2.1 & 594 & 609 & 7.1 \\
\hline 575 & & $\begin{array}{l}\text { Lewsi Drain at mouth, } \mathrm{SE}_{\frac{1}{4}} \\
\text { sec. } 31 \text {, T. } 3 \text { N., R.13 E. }\end{array}$ & 8.58 & 0 & 2.9 & 589 & 609 & 6.9 \\
\hline 576 & & $\begin{array}{l}\text { Gloede Ditch at Lewis Drain, } \\
\text { SE } \frac{1}{4} \text { sec. } 31 \text {, T. } 3 \text { N., R.13 E. }\end{array}$ & 15.9 & 2.0 & 5.4 & 589 & 643 & 10.0 \\
\hline 577 & & $\begin{array}{l}\text { Gloede Ditch above Feiblecorn } \\
\text { Drain, (gage 04-1652), SW sec.32, } \\
\text { T.3 N., R.13 E. }\end{array}$ & 16.0 & 1.4 & 6.0 & 588 & 643 & 9.2 \\
\hline 578 & & $\begin{array}{l}\text { Feiblecorn Drain at mouth, } \\
\text { SW } \frac{1}{4} \text { sec. } 32 \text {, T. } 3 \text { N., R.13 E. }\end{array}$ & .52 & 0 & 1.3 & 588 & 600 & 9.2 \\
\hline 579 & & $\begin{array}{l}\text { Gloede Ditch at Feiblecorn } \\
\text { Drain (at Hall Road), SW } \frac{1}{4} \text { sec. } \\
\text { 32, T. } 3 \text { N., R.13 E. }\end{array}$ & 16.5 & 1.4 & 6.0 & 588 & 643 & 9.2 \\
\hline 580 & & $\begin{array}{c}\text { Gloede Ditch above Utica } \\
\text { Drain, NW } \frac{1}{4} \text { sec.5, T.2 N., R.13 E. }\end{array}$ & 16.6 & 1.1 & 6.3 & 588 & 643 & 8.7 \\
\hline 581 & & $\begin{array}{l}\text { Utica Drain above Daus } \\
\text { Drain, Nh } \frac{1}{4} \text { sec.6, T.2 N., R.13 } \\
\text { E. }\end{array}$ & 1.90 & .9 & 3.3 & 596 & 640 & 13.3 \\
\hline
\end{tabular}


PHYSIOGRAPHIC DATA FOR SELECTED LOCATIONS IN THE CLINTON RIVER BASIN--CONTINUED

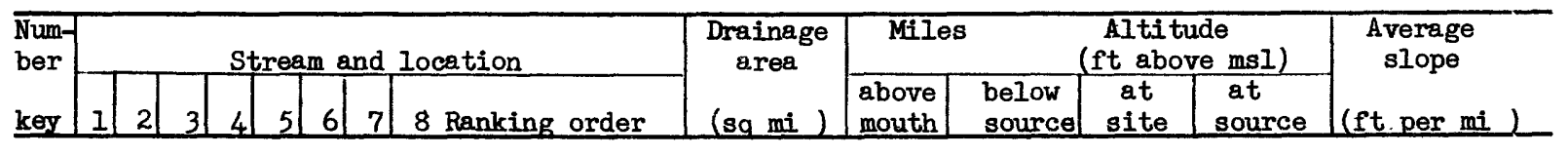

582

583

584

585

586

587

538

509

591

592

394

595

596

597

598

559
Daus Drain at mouth, NWt sec.6, T.2 N., R.13 E.
1.270
2.6
596
623
10.4

$\begin{array}{llllll}3.17 & .9 & 3.3 & 596 & 640 & 13.3\end{array}$
Drain, NWt sec.6, T.2 N., R.13 E.

Utica Drain above Kenner Drain, NEt sec.6, T.2 N., R.13 E.

$\begin{array}{llllll}3.35 & .3 & 3.9 & 590 & 640 & 12.8\end{array}$

Kenner Drain at mouth, NEt sec.6, T.2 N., R.13 E.

Utica Drain at Kenner Drain, NEt sec.6, T.2 N., R.13 E.

$$
\begin{aligned}
& \text { Utica Drain at mouth, Nht } \\
& \text { sec.5, T.2 N., R.13 E. }
\end{aligned}
$$

Gloede Ditch at Utica Drain, Nht sec.5, T.2 N., R.ì; E。

Gloede Ditoh above Crittenden Drain, Nht sec.5, T.2 N., R.13 E。

$$
\text { Crittenden Drain at mouth, }
$$

$\mathrm{NW}_{4}^{\frac{3}{4}}$ sec.5, T.2 N., R.13 E.

Gloede Ditch at Crittenden

Drain, Nwt sec.5, T.2 N.,

R.13 E。

Glcede Ditch at mouth, SEd sec.5, T.2 N., R.13 E。

Midale Branch Clinton River at Gloede Ditch, SEt sec.5, T.2

N. R. R E.

Midule Branch Clinton River above

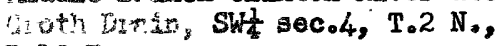
[..13 E.

\footnotetext{
Groth Mria it mouth, SW

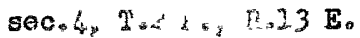

Midid. stars Oisuton River at

Groti beatin, gite sec.4, T.2

No, R.I?

$\begin{array}{llllll}.92 & 0 & 1.6 & 590 & 605 & 9.4\end{array}$

$\begin{array}{llllll}4.27 & .3 & 3.9 & 590 & 640 & 12.8\end{array}$

$\begin{array}{llllll}4.40 & 0 & 4.2 & 588 & 640 & 12.4\end{array}$

$\begin{array}{llllll}21.0 & 1.1 & 6.3 & 588 & 643 & 8.7\end{array}$

$\begin{array}{llllll}21.0 & .8 & 6.6 & 537 & 643 & 8.5\end{array}$

$\begin{array}{llllll}2.22 & 0 & 6.1 & 587 & 633 & 7.5\end{array}$

$\begin{array}{llllll}23.3 & .8 & 6.6 & 587 & 643 & 8.5\end{array}$

$\begin{array}{llllll}23.5 & 0 & 7.4 & 581 & 643 & 8.4\end{array}$

$\begin{array}{llllll}72.5 & 2.2 & 18.6 & 581 & 818 & 12.7\end{array}$

$\begin{array}{llllll}72.6 & 2.0 & 18.8 & 580 & 818 & 12.7\end{array}$

$\begin{array}{llllll}.61 & 0 & 1.2 & 580 & 595 & 12.5\end{array}$

$\begin{array}{llllll}73.2 & 2.0 & 18.8 & 580 & 818 & 12.7\end{array}$

Midale bruch ainton Rivor gbove 74.' $\quad .4 \quad 20.4 \quad 578 \quad 818 \quad 21.8$

Miliar Drein, pripate clain 546,

T.2 N., R. $13 \mathrm{E}$.

Miller biain above Bobuean

Drain, ing sec.27, T.3 î.

$.85 \quad 4.5 \quad 3.0 \quad 596 \quad 605$

3.0 R. .3 .3 .

Eucean Druin at moutr,

Net. se.. $27, T_{2} 3 \mathrm{~N}, \mathrm{R}_{0.13 \mathrm{E}}$

$.74 \quad 0$

.8

596

600

5.0 
PHYSIOGRAPHIC DATA FOR SEUECTED LOCATIONS IN THE CLTNTON RIVER BASIN-CONTINUED

\begin{tabular}{|c|c|c|c|c|c|c|c|c|c|c|c|c|c|c|}
\hline $\begin{array}{l}\text { Num- } \\
\text { ber }\end{array}$ & & & & & rea & $m a$ & ind & location & $\begin{array}{c}\text { Drainage } \\
\text { area }\end{array}$ & Mil & & $\begin{array}{r}\mathrm{Al} \\
\text { (ft a }\end{array}$ & $\begin{array}{l}\text { itude } \\
\text { ove msl) }\end{array}$ & $\begin{array}{l}\text { A verage } \\
\text { slope }\end{array}$ \\
\hline key & 1 & 2 & 3 & 4 & 5 & 6 & 7 & 8 Ranking order & $(\mathrm{sg} \mathrm{mi})$ & $\begin{array}{l}\text { above } \\
\text { mouth }\end{array}$ & $\begin{array}{l}\text { below } \\
\text { source }\end{array}$ & $\begin{array}{l}\text { at } \\
\text { site }\end{array}$ & $\begin{array}{c}\text { at } \\
\text { source }\end{array}$ & (ft per mi ) \\
\hline
\end{tabular}

600

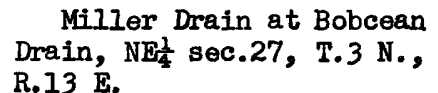

601 Miller Drain above Nicol
Drain, private claim 610
T.2 N., R.13 E.

602

603 Nicol Drain at mouth
private claim 610, T.2 N., R. $13 \mathrm{E}$.

604

605

606

607 Miller Drain at Nicol Drain private claim 610, T.2 N., R. $13 \mathrm{E}$. Miller Drain at Heydenreich Road, private claim 546, T.2 N., R.13 E.

$4.5 \quad 3.0$

2.98

1.4

6.1

.790

2.0

3.77

1.46 .1

$4 \cdot 50$

$$
.2
$$

7.3

$4.58 \quad 0$

\section{5}

578

589

600 claim 546, T.2 N., R.13 E.

Middle Branch Clinton River at Miller Drain, private claim

79.3 546, T.2 N., R.13 E. Middle Branch Clinton' River at mouth, private claimi 546, T.2 N., R.13 E.

608

North Branch Clinton River at Middle 281 Branch Clinton River, private claim 546, T.2 N., R.13 E.

609

North Branch Clinton River at mouth 281 private claim 546, T.2 N., R.13 E.

610

Clinton River at North Branch Clinton 734 River, private claim 546, T.2 N., R.13 E.

611 Clinton River at Moravian Drive (gage 04-1655), private claim 546, T.2 N., R. $13 \mathrm{E}$.

612 Clinton River above Harrington Drain, 735 private claim 137, T.2 No, R。 $13 \mathrm{E}$.

6.3 Harrington Drain above Schroeder Drain, SE $\frac{1}{4}$ sec.I, T.I N., R.12 E.

34 Schrooder Drain at mouth, $\mathrm{SE}_{4} \frac{1}{4}$ sec.I, T.I No, R.I2 E。

6 Harrington Drain at Schroeder Drain, $\mathrm{SEF}_{4}^{\frac{1}{4}}$ sec.1, T.1 N., R.12 E。 $\begin{array}{llllll}6.47 & 6.4 & 5.0 & 604 & 624 & 4.0\end{array}$

$\begin{array}{llllll}1.10 & 0 & 1.7 & 604 & 613 & 5.3\end{array}$

$\begin{array}{llllll}7.57 & 6.4 & 5.0 & 604 & 624 & 4.0\end{array}$ 
PHYSIOGRAPHIC DATA FOR SEIECTED LOGATIONS IN THE CLINTON RIVER BASIN-CONTINUED

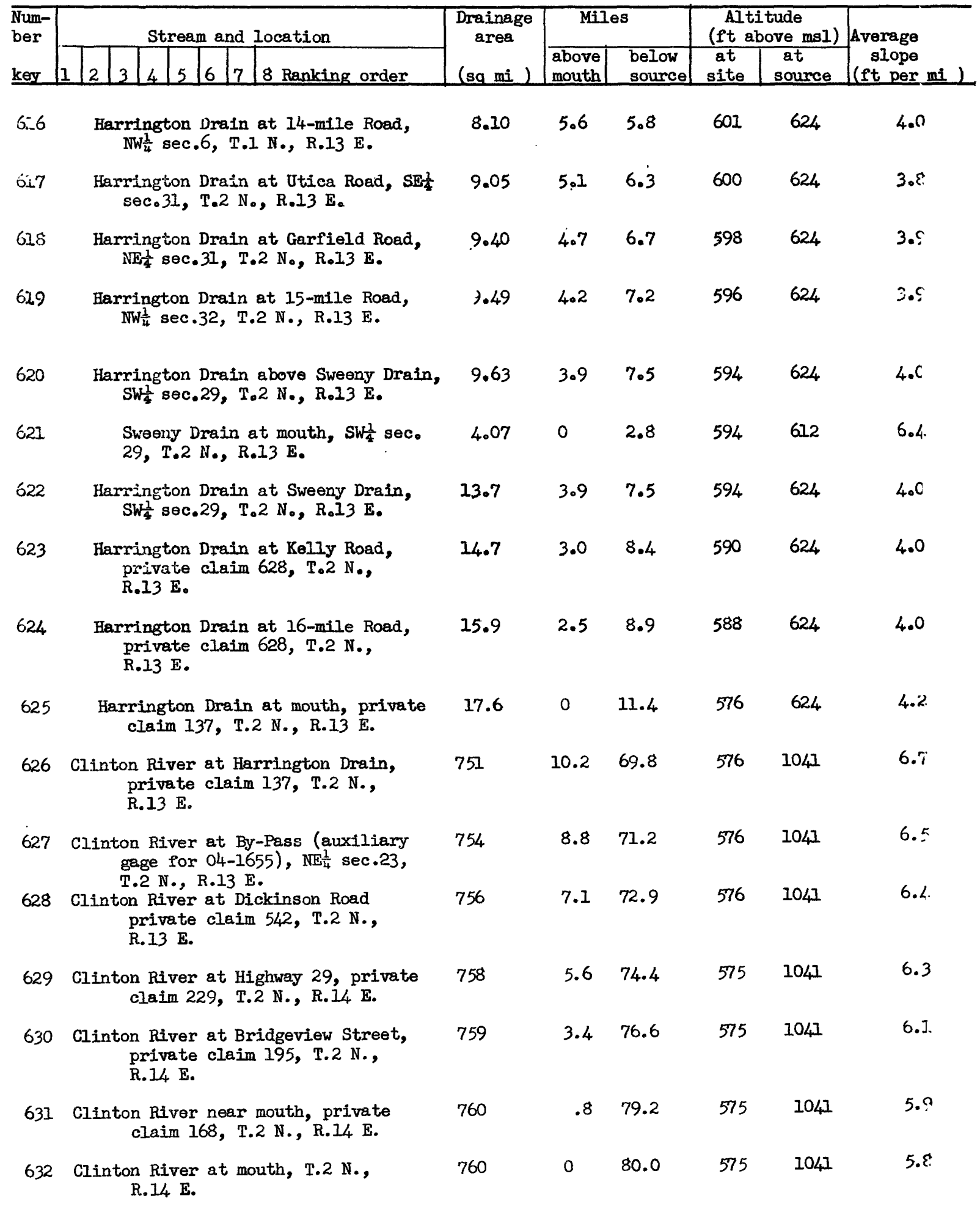

